\title{
THE PROPERTIES OF THE LOCAL INTERSTELLAR MEDIUM AND THE INTERACTION OF THE STELLAR WINDS OF $\epsilon$ INDI AND $\lambda$ ANDROMEDAE WITH THE INTERSTELLAR ENVIRONMENT ${ }^{1}$
}

\author{
BRIAN E. WOOD \\ JILA, University of Colorado, Boulder, CO 80309-0440; wood@marmot.colorado.edu \\ William R. Alexander \\ 1250 Buffalo Creek Road, Huntington, WV 25704-9608; walexander@ashland.e-mail.com \\ AND \\ JEFFREY L. LINSKY \\ JILA, University of Colorado, Boulder, CO 80309-0440;jlinsky@jila.colorado.edu \\ Received 1995 November 27; accepted 1996 May 6
}

\begin{abstract}
We present new observations of the Ly $\alpha$ lines of $\epsilon$ Indi (K5 V) and $\lambda$ Andromedae (G8 IV-III + ?). These data were obtained by the Goddard High Resolution Spectrograph (GHRS) on the Hubble Space Telescope. Analysis of the interstellar H I and D I absorption lines reveals that the velocities and temperatures inferred from the $\mathrm{H}$ I lines are inconsistent with the parameters inferred from the $\mathrm{D}_{\mathrm{I}}$ lines, unless the $\mathrm{H}$ I absorption is assumed to be produced by two absorption components.

One absorption component is produced by interstellar material. For both lines of sight observed, the velocity of this component is consistent with the velocity predicted by the local flow vector. For the $\epsilon$ Ind data, the large velocity separation between the stellar emission and the interstellar absorption allows us to measure the $\mathrm{H} \mathrm{I}$ column density independent of the shape of the intrinsic stellar Ly $\alpha$ profile. This approach permits us to quote an accurate column density and to assess its uncertainty with far more confidence than in previous analyses, for which the errors were dominated by uncertainties in the assumed stellar profiles. For the short $(d=3.46 \mathrm{pc})$ line of sight to $\epsilon$ Ind, the $\mathrm{H}$ I column density is found to be $\log N_{\mathrm{HI}}=18.0 \pm 0.1$, which implies an average density for the local interstellar medium (LISM) of $n_{\mathrm{HI}}=0.094 \pm 0.022 \mathrm{~cm}^{-3}$. For the much longer $(d=23 \mathrm{pc})$ line of sight to $\lambda$ And, we estimate the $\mathrm{H}_{\mathrm{I}}$ column density to be $\log N_{\mathrm{HI}}=18.45 \pm 0.15$, which corresponds to an average density of $n_{\mathrm{HI}}=$ $0.041 \pm 0.014 \mathrm{~cm}^{-3}$. The $\mathrm{D} / \mathrm{H}$ ratios we measure from the data are $(1.6 \pm 0.4) \times 10^{-5}$ and $(1.7 \pm 0.5) \times 10^{-5}$ for $\epsilon$ Ind and $\lambda$ And, respectively. These values are consistent with those measured from observations of Capella, Procyon, and $\alpha$ Cen. We measure LISM temperatures of $T=8500 \pm 500$ $\mathrm{K}$ and $T=11,500 \pm 500 \mathrm{~K}$ from the $\epsilon$ Ind and $\lambda$ And data, respectively. The $\lambda$ And temperature is significantly higher than temperatures previously measured from GHRS data, which leads us to speculate that the $\mathrm{H}$ I and $\mathrm{D}_{\mathrm{I}}$ absorption lines may be broadened by multiple ISM components with different velocities. The results of our $\lambda$ And analysis should be considered as tentative, until GHRS observations of the much narrower $\mathrm{Mg}$ II and/or Fe II absorption lines can be obtained.

We believe that hot hydrogen surrounding $\epsilon$ Ind and $\lambda$ And is responsible for the second $\mathrm{H}$ i absorption component, although we consider this conclusion to be tentative in the case of $\lambda$ And. These "hydrogen walls" are produced by the interaction of the winds of these stars with the surrounding interstellar material. An anologous solar hydrogen wall has been predicted by recent models of the heliospheric interface region and confirmed by GHRS observations of $\alpha$ Cen. The column densities we measure for the second components are $\log N_{\mathrm{H} 1}=14.2 \pm 0.2$ and $\log N_{\mathrm{HI}}=14.8 \pm 0.2$ for $\epsilon$ Ind and $\lambda$ And, respectively, and the temperatures are $100,000 \pm 20,000 \mathrm{~K}$ and $62,000 \pm 18,000 \mathrm{~K}$. These temperatures are too hot for the solar hydrogen wall, and for $\epsilon$ Ind the velocity of the second component is clearly inconsistent with the solar hydrogen wall. Thus, for these components we assume a stellar origin, in which the higher temperatures are a consequence of higher interstellar wind velocities in the stellar rest frames. Because the heliospheric models demonstrate the importance of the solar wind in the formation of the solar hydrogen wall, our detection of anologous structure around $\epsilon$ Ind and perhaps $\lambda$ And may constitute a first detection of solar-like winds around dwarf and subgiant stars.
\end{abstract}

Subject headings: ISM : abundances - ISM: kinematics and dynamics --

stars: individual ( $\epsilon$ Indi, $\lambda$ Andromedae) - stars: mass loss - ultraviolet: stars

\footnotetext{
${ }^{1}$ Based on observations with the NASA/ESA Hubble Space Telescope, obtained at the Space Telescope Science Institute, which is operated by the Association of Universities for Research in Astromomy Inc., under NASA Contract NAS 5-26555.
} 


\section{INTRODUCTION}

The Goddard High Resolution Spectrograph (GHRS) aboard the Hubble Space Telescope (HST) is an invaluable tool in the study of the local interstellar medium (LISM) because it is the first instrument capable of fully resolving narrow interstellar absorption features in the ultraviolet. The first study of the LISM using GHRS observations was that of Linsky et al. (1993, hereafter Paper I), who determined the properties of the LISM along the $12.5 \mathrm{pc}$ line of sight to Capella. The focus of their analysis was on the abundance ratio of deuterium to hydrogen (i.e., the $\mathrm{D} / \mathrm{H}$ ratio). This ratio can be used to estimate the primordial $\mathrm{D} / \mathrm{H}$ ratio, which is a very important quantity for cosmology because it is the most sensitive diagnostic available for estimating the baryon density of the universe (see, e.g., Walker et al. 1991). The $\mathrm{D} / \mathrm{H}$ ratio for the Capella line of sight was found to be $1.65_{-0.18}^{+0.07} \times 10^{-5}$ (Paper I). An analysis of more recent GHRS observations of Capella led Linsky et al. (1995, hereafter Paper II) to slightly alter this value to $1.60_{-0.19}^{+0.14} \times 10^{-5}$. The errors quoted here are a combination of the random errors and the estimated systematic errors.

In Paper II, GHRS observations of ISM absorption lines seen along the line of sight toward Procyon $(d=3.5 \mathrm{pc})$ were also analyzed. Unfortunately, the echelle-A grating was not available at the time of these observations, and it was therefore necessary to use the lower resolution G160M grating. This complicated the analysis because the ISM lines were not fully resolved. Another complicating factor was a second ISM absorption component, which is separated by only $2.6 \mathrm{~km} \mathrm{~s}^{-1}$ from the component associated with the local cloud. An estimate of $\mathrm{D} / \mathrm{H}=(1.4 \pm 0.2) \times 10^{-5}$ was given in Paper II, which is consistent with the Capella result. It was also shown that a resonable alteration of the assumed stellar Ly $\alpha$ line yields a $\mathrm{D} / \mathrm{H}$ value even closer to the Capella result. Lemoine et al. (1995) found three ISM absorption components in their analysis of the ISM along the line of sight toward the white dwarf G191-B2B, which is only $7^{\circ}$ away from Capella. One of the three components detected toward G191-B2B matched the absorption component detected for the Capella line of sight very well in terms of velocity, temperature, and column density. However, the value of $\mathrm{D} / \mathrm{H}$ for this component derived by Lemoine et al. (1995) on the basis of their G160M observations of $\mathrm{Ly} \alpha, \mathrm{D} / \mathrm{H}=1.4_{-0.3}^{+0.1} \times 10^{-5}$, is slightly lower than the value derived in Paper II.

In addition to measuring the local $\mathrm{D} / \mathrm{H}$ ratio, these analyses also sought to investigate the properties of the LISM. Using measurements of optical Ca II LISM absorption lines observed for many lines of sight, Lallement \& Bertin (1992) determined two different flow vectors for local interstellar material. For lines of sight in the general direction of the Galactic center, the flow vector points toward $l=184^{\circ} .5$ and $b=-20.5$ with a velocity of $v_{0}=29.4 \mathrm{~km}$ $\mathrm{s}^{-1}$, whereas the flow vector for lines of sight in roughly the anti-Galactic center direction points toward $l=186.1$ and $b=-16.4$ with a velocity of $v_{0}=25.7 \mathrm{~km} \mathrm{~s}^{-1}$. These two vectors are referred to as the $V_{\mathrm{G}}$ and $V_{\mathrm{AG}}$ vectors, respectively, and the interstellar clouds they represent are the $G$ and AG clouds. The latter is also called the Local Interstellar Cloud (LIC). Since the $V_{\mathrm{G}}$ and $V_{\mathrm{AG}}$ vectors are very similar, it is debatable whether the $G$ and $A G$ clouds are really separate entities. Nevertheless, there is evidence that
LISM material in the Galactic center direction is moving slightly faster than LISM material in the opposite direction. Other ISM absorption components have been observed toward very nearby stars that are not consistent with either the $V_{\mathrm{G}}$ or the $V_{\mathrm{AG}}$ vectors. For example, besides the aforementioned second component seen in the Procyon data, Lallement et al. (1994) observed a second ISM absorption component for the even shorter $2.7 \mathrm{pc}$ line of sight toward Sirius.

The Ulysses satellite measured a velocity of $26 \pm 1 \mathrm{~km}$ $\mathrm{s}^{-1}$ for interstellar He I atoms in the solar system (Witte et al. 1993). This velocity agrees much better with the modulus of the $V_{\mathrm{AG}}$ vector $\left(25.7 \mathrm{~km} \mathrm{~s}^{-1}\right)$ than the modulus of the $V_{\mathrm{G}}$ vector $\left(29.4 \mathrm{~km} \mathrm{~s}^{-1}\right)$, suggesting that the former represents the true local cloud. The GHRS observations of Capella, Procyon, G191-B2B, Sirius, and other stars (see, e.g., Gry et al. 1995; Ferlet et al. 1995; Lallement et al. 1995) have also established the $V_{\mathrm{AG}}$ vector as the flow vector that best characterizes the interstellar material in the immediate vicinity of the Sun. As summarized by Lallement et al. (1995), nearly all of the lines of sight observed by the GHRS so far show interstellar absorption at the velocity predicted by the $V_{A G}$ vector, and almost none shows absorption at the velocity predicted by the $V_{\mathrm{G}}$ vector. The exception is the $1.3 \mathrm{pc}$ line of sight to $\alpha \mathrm{Cen}$, which ironically is the nearest star system to the Sun and therefore is the line of sight that one would think would best represent the properties of the ISM in the immediate solar vicinity. The interstellar material detected along the $\alpha$ Cen line of sight appears to be entirely in the $G$ cloud. If the $V_{\mathrm{AG}}$ vector really does best represent the local cloud, it is very curious that no absorption is seen at the velocity predicted by this vector. Lallement et al. (1995) interpreted this to mean that the boundary between the $G$ and AG clouds is very nearby for the Sun- $\alpha$ Cen line of sight.

In a thorough analysis of GHRS echelle observations of interstellar Mg II, Fe II, H I, and D I absorption lines seen toward $a$ Cen, Linsky \& Wood (1996, hereafter Paper III) found that the temperature and abundances of the material along the $\alpha$ Cen line of sight are different from those seen toward Procyon and Capella. The temperature and nonthermal velocity found for the Capella line of sight are $T=7000 \pm 900 \mathrm{~K}$ and $\xi=1.6 \pm 0.6 \mathrm{~km} \mathrm{~s}^{-1}$, respectively (Paper II). Similar values were found for the Procyon line of sight $\left(T=6900 \pm 380 \mathrm{~K}\right.$ and $\xi=1.21 \pm 0.27 \mathrm{~km} \mathrm{~s}^{-1}$; Paper II). These quantities include estimates of systematic errors. Although the nonthermal velocities detected toward $\alpha$ Cen $\left(\xi=1.20 \pm 0.25 \mathrm{~km} \mathrm{~s}^{-1}\right)$ are similar to the Capella and Procyon line-of-sight values, the temperature reported in Paper III is significantly lower $(T=5400 \pm 500 \mathrm{~K})$.

The quantity used in Paper III to demonstrate the difference between the LISM abundances along the $\alpha$ Cen line of sight and the abundances observed toward Capella and Procyon is the $\mathrm{D} \mathrm{I}_{1} / \mathrm{Mg}$ II ratio. This ratio is $4.1 \pm 0.4$ and $5.1 \pm 0.7$ toward Capella and Procyon, respectively (Paper II), but toward $\alpha$ Cen the ratio is much lower $(1.2 \pm 0.2$; Paper III). The $\mathrm{D}_{\mathrm{I} / \mathrm{Fe}}$ II ratio shows a similar behavior. This proves that abundances vary significantly in the LISM over distance scales of only a few parsecs. In order to determine whether it is $\mathrm{D} / \mathrm{H}, \mathrm{Mg} / \mathrm{H}$ (and $\mathrm{Fe} / \mathrm{H}$ ), or both that are varying, it is necessary to measure accurately the $\mathrm{H} \mathrm{I}$ column densities for all these stars to calculate the absolute abundances. Unfortunately, this proved to be impossible for the $\alpha$ Cen line of sight. 
The analysis of the $\alpha$ Cen line of sight in Paper III provides a dramatic illustration of the difficulties in measuring interstellar $\mathrm{H}$ i column densities from the very broad, saturated absorption features present in $L y \alpha$ lines. The profiles of the $\mathrm{Mg}$ II, Fe II, and D I absorption lines show no evidence for more than one absorption component, but when the H I Ly $\alpha$ absorption line was fitted with only one component, it was found that the velocity and temperature measured from this line were inconsistent with the other lines. The only way to resolve this discrepancy was to add a second absorption component to the $\mathrm{H}_{\mathrm{I}}$ line, one with a column density too low to be detected in the other lines. Although the single-component fits to the $\mathrm{H}$ I absorption observed toward $\alpha$ Cen A and B were shown to be unique solutions, the two-component fits were not. By modifying the assumed stellar Ly $\alpha$ profiles used for $\alpha$ Cen A and B in a reasonable way, we found acceptable fits to the observed profiles with very different values for the interstellar $\mathrm{H}_{\mathbf{I}}$ column density, leading to a very large uncertainty in this quantity $\left(\log N_{\mathrm{HI}}=17.8 \pm 0.3\right)$.

The additional absorption component was found to have a very interesting interpretation. Recent models of the heliospheric interface region (shown schematically in Fig. 1), where the outflowing solar wind collides with the interstellar wind, predict a region of heated, compressed, and decelerated $\mathrm{H}$ I just outside the heliopause (see, e.g., Baranov \& Malama 1995; Pauls, Zank, \& Williams 1995).
This region, shown as a shaded area in Figure 1, has been referred to as a "hydrogen wall." The temperature, column density, and flow velocity of the hydrogen wall predicted by the models agree nicely with the parameters of the second $\mathrm{H}$ I absorption component detected toward $\alpha$ Cen, which suggests that the hydrogen wall has been detected, thereby supporting the models of the theorists. After taking into account the effects that a similar hydrogen wall around $\alpha$ Cen might have on the analysis, the temperature and column density of the solar hydrogen wall along the $\alpha$ Cen line of sight were estimated to be $T=29,000 \pm 5000 \mathrm{~K}$ and $\log N_{\mathrm{HI}}=14.74 \pm 0.24$, respectively (Paper III).

With all this in mind, we now present our analysis of the $\mathrm{H}$ I and D I absorption features detected in the Ly $\alpha$ lines of $\epsilon$ Indi and $\lambda$ Andromedae. Besides investigating the properties of the LISM with these data, we also hope to learn more about the solar hydrogen wall and/or detect analogous hydrogen walls around $\epsilon$ Ind and $\lambda$ And.

\section{GHRS OBSERVATIONS OF $\epsilon$ INDI AND $\lambda$ ANDROMEDAE}

The two targets we have studied with the GHRS were previously observed with the Copernicus and IUE satellites in order to analyze the properties of the LISM along these lines of sight (McClintock et al. 1978; Baliunas \& Dupree 1979; Murthy et al. 1990). The K5 V star $\epsilon$ Ind (= HD 209100 ) is located only $3.46 \mathrm{pc}$ from the sun at Galactic coordinates $l=336^{\circ}$ and $b=-48^{\circ}$ (Gliese \& Jahreiss

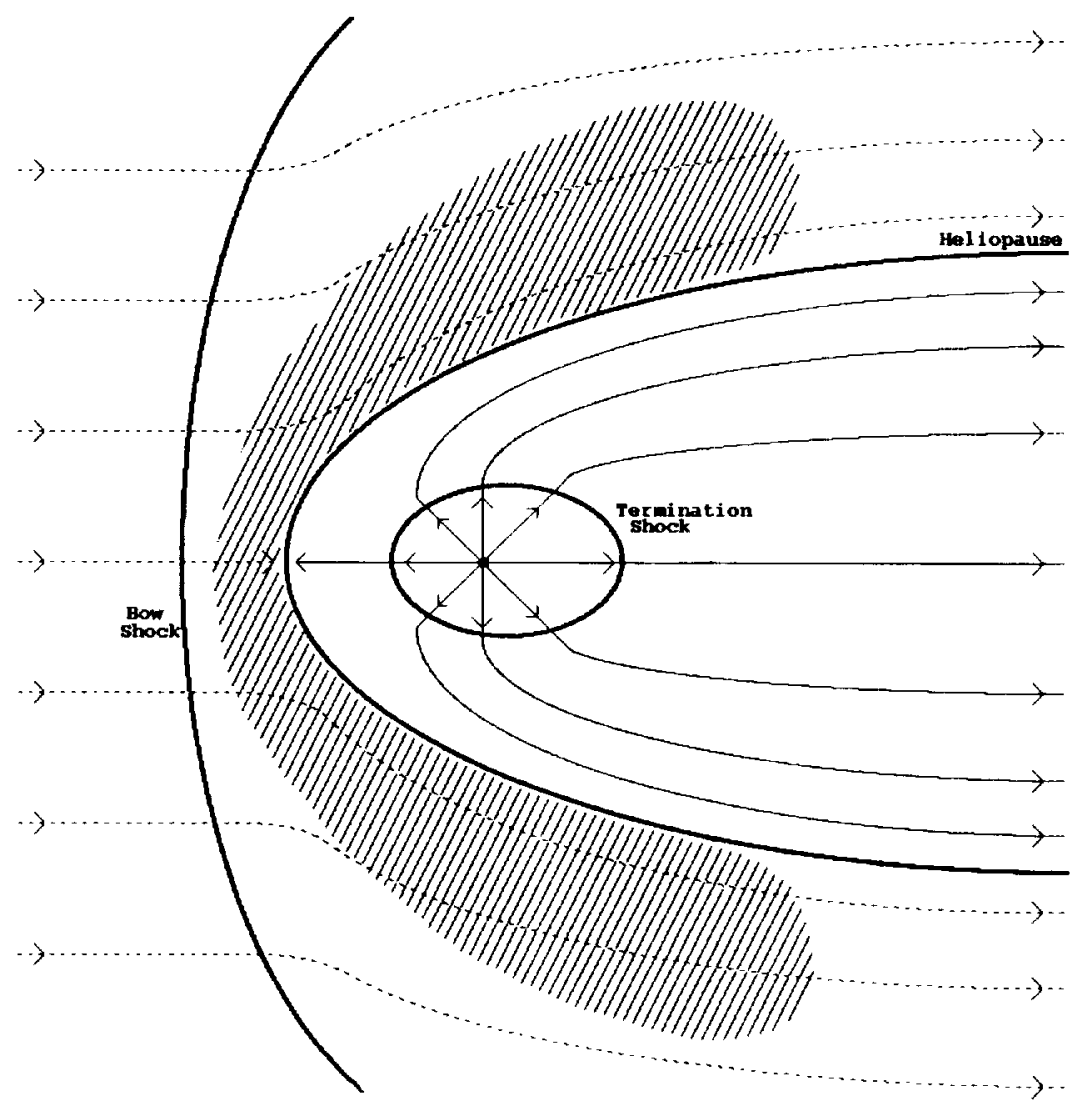

Fig. 1.-Schematic illustration of how the solar wind (thin solid lines) interacts with the plasma component of the interstellar wind (dotted lines). The solar wind is decelerated from supersonic speeds to subsonic speeds at the termination shock. The bow shock is a standing shock wave through which the supersonic interstellar wind passes. The heliopause is the contact surface separating the plasma flows of the solar and insterstellar winds. Some neutral hydrogen in the interstellar wind is deflected around the heliopause with the protons, but some also penetrates into the heliosphere. The shaded area is a region of heated, compressed, and decelerated $\mathrm{H} 1$ that has been referred to as a "hydrogen wall." The hydrogen wall is roughly $150 \mathrm{AU}$ from the Sun in the upwind direction according to recent models of the heliosphere (Baranov \& Malama 1995; Pauls et al. 1995). 
TABLE 1

SUMMARY OF GHRS OBSERVATIONS

\begin{tabular}{|c|c|c|c|c|c|c|c|}
\hline Target & Grating & $\begin{array}{l}\text { Aperture and } \\
\text { Substep Pattern }\end{array}$ & $\begin{array}{c}\text { Spectral Range } \\
(\AA)\end{array}$ & $\begin{array}{l}\text { Spectral Resolution } \\
\left(\mathrm{km} \mathrm{s}^{-1}\right)\end{array}$ & $\begin{array}{l}\text { Exposure Time } \\
\text { (s) }\end{array}$ & Date & $\begin{array}{l}\text { Start Time } \\
\text { (UT) }\end{array}$ \\
\hline$\lambda$ And ....... & EA-46 & SSA 9 & $1212-1219$ & 3.57 & 3318 & 1994 Aug 31 & $15: 18$ \\
\hline$\epsilon$ Ind.. & EA-46 & SSA 9 & 12121219 & 3.57 & 3291 & 1995 Mar 18 & $2: 42$ \\
\hline
\end{tabular}

1991). The $\lambda$ And (=HD 222107) star system is an RS CVn binary system about 23 pc away (Jenkins 1952) with a 20.52 day orbital period (Batten, Fletcher, \& Mann 1978), consisting of a G8 IV III star and a companion star of unknown spectral type (Herbst 1973; Strassmeier et al. 1988). The Galactic coordinates of $\lambda$ And are $l=110^{\circ}$ and $b=-15^{\circ}$.

Table 1 summarizes the relevant information about our observations of the Ly $\alpha$ lines of $\epsilon$ Ind and $\lambda$ And. Images of the Pt-Ne calibration lamp were taken prior to both observations and were used to calibrate the wavelengths of the spectra. The spectra displayed in Figure 2 show very broad absorption lines centered near $1215.6 \AA$ that are due to interstellar H I. Located about $-0.33 \AA$ from the $\mathrm{H}_{\mathrm{I}}$ absorption lines are the narrower $\mathrm{D}$ I absorption lines. The emission feature at $1215.74 \AA$ in the $\epsilon$ Ind spectrum is due to geocoronal $\mathrm{H}$ I. In our analysis, we corrected for this emission by fitting a Gaussian to the feature and then subtracting the Gaussian from the data.

The cores of the $\mathrm{HI}$ absorption lines should have zero flux because of the interstellar absorption, but our spectra show positive flux there, which is caused by scattered light (see Papers I and III). Because the scattered light level is constant in the saturated line cores, we correct for this flux

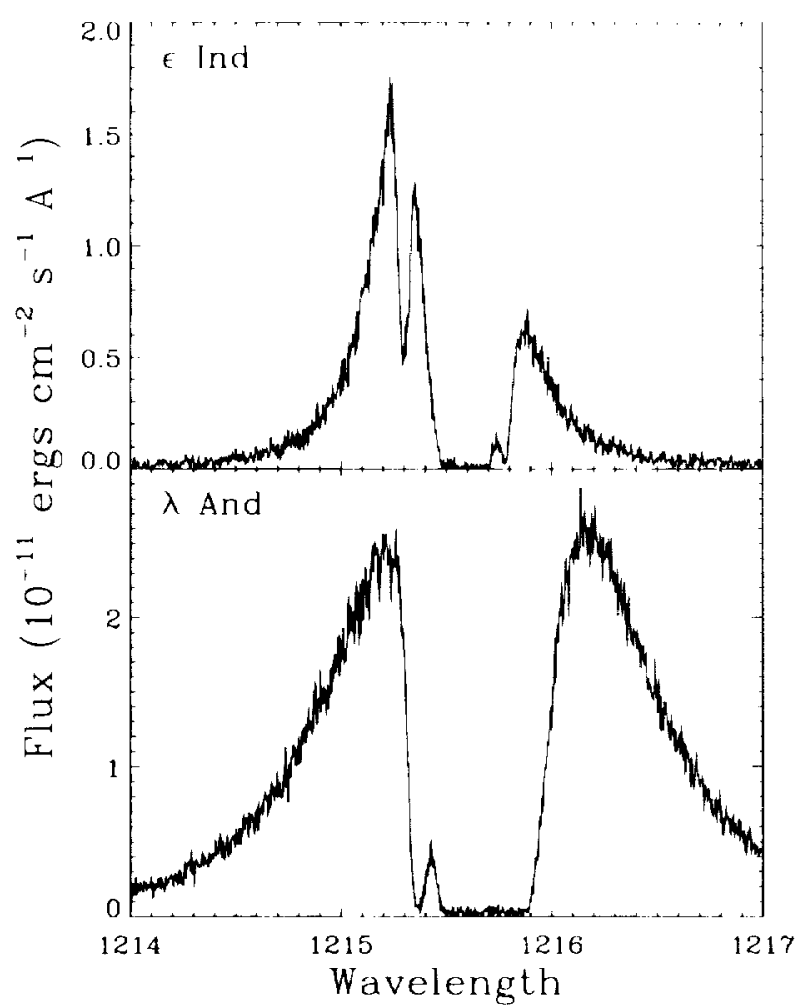

FIG. 2.-GHRS observations of the Ly $\alpha$ lines of $\epsilon$ Ind and $\lambda$ And, showing broad interstellar $\mathrm{H}_{\mathrm{I}}$ absorption at $1215.6 \AA$ and narrow $\mathrm{D}$ I absorption near $1215.3 \AA$. The emission feature at $1215.74 \AA$ in the $\epsilon$ Ind spectrum is geocoronal emission. by simply subtracting the mean flux level observed in the line cores $\left(1.2 \times 10^{-13}\right.$ and $3.1 \times 10^{-13} \mathrm{ergs} \mathrm{cm}^{-2} \mathrm{~s}^{1} \AA$ for $\epsilon$ Ind and $\lambda$ And, respectively) from a spectral region (1215.2-1215.85 $\AA$ and $1215.2-1216.1 \AA$ for $\epsilon$ Ind and $\lambda \hat{A}$. respectively) encompassing the absorption. In all figures except Figure 2, the spectra displayed will be the observed spectra after the corrections for the scattered light and geocoronal emission have been applied.

\section{SINGLE-COMPONENT FITS TO THE DATA}

The first step in analyzing the line profiles is to obtain initial estimates for the intrinsic stellar Ly $\alpha$ emission lines. Because $\epsilon$ Ind has a spectral type $(\mathrm{K} 5 \mathrm{~V})$ similar to $\alpha$ Cen $B$ ( $\mathrm{K} 1 \mathrm{~V}$ ) and both are inactive stars, we adopt the Ly $\alpha$ profile used for $\alpha$ Cen B in Paper III (profile model 1B, to be specific) as our first estimate for the line profile of $\epsilon$ Ind. We normalize the fluxes of this profile so that the model fits the observed profile in the far wings of the Ly $\alpha$ line.

Following a procedure similar to that used in Paper III, we use a $\chi^{2}$ minimization technique to determine the best single-component fit to the $\mathrm{D}_{\mathrm{I}}$ and $\mathrm{H}_{\mathrm{I}}$ absorption lines. The atomic data necessary for the computation are laken from Morton (1991). Throughout this paper, we correct for instrumental broadening using a Gaussian with FWHM $=3.7$ pixels as the instrumental profile (Gilliland 1994). Because the D I and $\mathrm{H}$ I absorption features are both fine-structure doublets, we include both components of the doublets in our computations. For $\alpha$ Cen $A$ and $B$, the D 1 and $H_{I}$ absorption lines are well separated, and it was not difficult to use a polynomial fit to estimate what the line profile would look like in absence of any D I absorption. Therefore, in Paper III the D I and $\mathrm{H}$ I lines were analyzed separately. We cannot do this for either $\epsilon$ Ind or $\lambda$ And because we do not believe that polynomial fits can accurately estimate the "continuum" upon which the $D_{\text {I }}$ absorption is superposed (see Fig. 2). Therefore, in this paper we must fit the $\mathrm{DI}$ and $\mathrm{H} I$ lines simultaneously.

The initial fit to the $\epsilon$ Ind data using the $\alpha$ Cen B profile model was not very good, so we used the residuals of that fit to alter the assumed stellar profile. This greatly improved the quality of the fit. After some more adjustments to the stellar profile, we obtained our best single-component fit 10 the $\epsilon$ Ind data, shown in Figure 3. The parameters of the fit are given in Table 2, including the line velocities, Doppler parameters $(b)$, logarithmic column densities $(\log N)$, and optical depths $(\tau)$ of the $\mathrm{H}_{\mathrm{I}}$ and $\mathrm{D}_{\mathrm{I}}$ absorption lines. The quality of all the fits in this paper can be assessed either by looking at the residuals displayed in the figures or by noting the $\chi_{*}^{2}$ values listed in the tables (see, e.g., Bevington $\&$ Robinson 1992). The errors given in Table 2 , which were estimated using Monte Carlo techniques, represent only the random errors in the fitting procedure and do not include the systematic errors that certainly dominate the uncertainty in the analysis. 


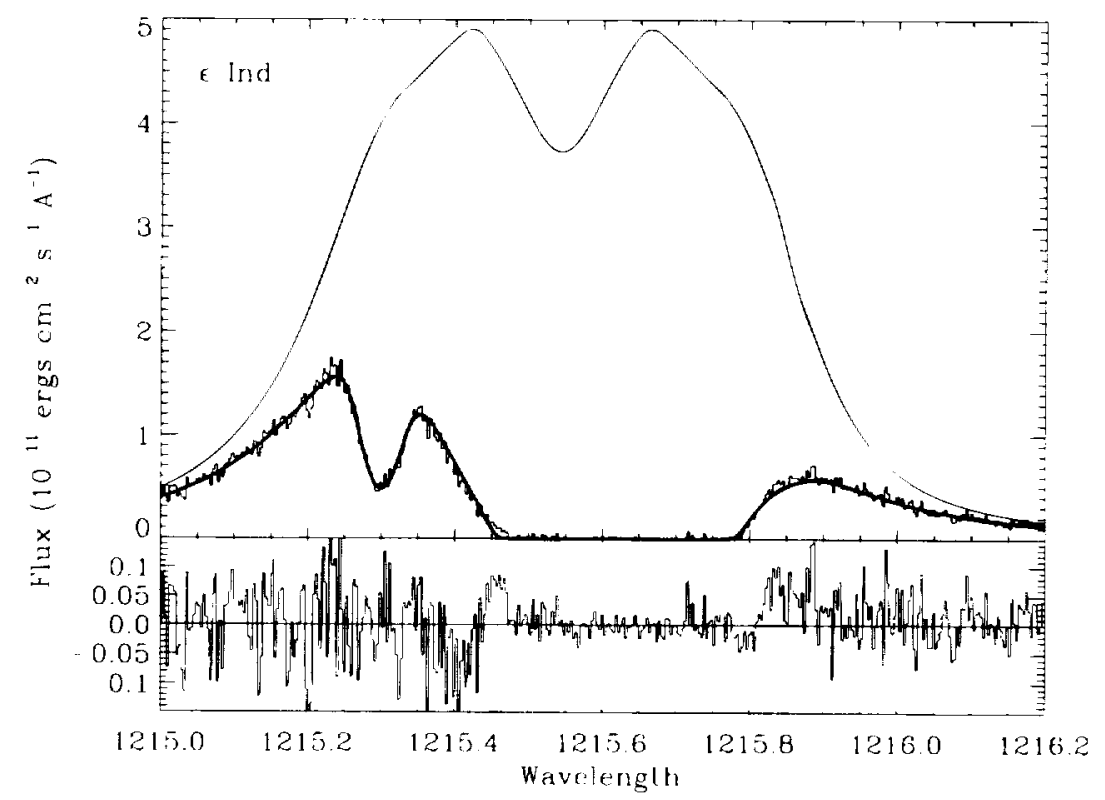

FIG. 3. - Our best single-component fit to the $\mathrm{H}_{\mathrm{I}}$ and $\mathrm{D}_{\mathrm{I}}$ absorption lines of $\epsilon$ Ind, with the residuals displayed below the fit. The data are shown in histogram form. The thin solid line is the assumed stellar Ly $\alpha$ profile, and the thick solid line is the fit. The parameters for the fit are given in Table 2.

For $\lambda$ And, we adopt as our initial estimate for the intrinsic stellar profile the Ly $\alpha$ profile used in Paper I for the G8 star of the Capella binary system. This is reasonable because the spectral type of $\lambda$ And (G8 IV-III) is very similar to that of the primary star of the Capella system (G8 III). We narrow the profile slightly to force the wings of the model profile to agree better with the shape of the wings of the observed Ly $\alpha$ profile, and then we fit the data as we did for $\epsilon$ Ind. Figure 4 shows our best single-component fit to the $\lambda$ And data. The parameters of the fit are given in Table 2 .

The residuals of the $\epsilon$ Ind fit in Figure 3 reveal systematic discrepancies between the fit and the data. Attempts to correct these discrepancies by altering the assumed stellar $\mathrm{Ly} \alpha$ profile resulted in unreasonable fine structure in the stellar profile. For example, to correct for the flux excess near $1215.47 \AA$ one must assume that the stellar profile flux near $1215.47 \AA$ is at least 3 times larger than the flux at $1215.45 \AA$. A flux increase this steep is not reasonable. The $\lambda$ And fit in Figure 4 is a much better fit than the $\epsilon$ Ind fit, but both suffer from another problem, namely that the parameters of the fits in Table 2 suggest that $\mathrm{D}_{\mathrm{I}}$ and $\mathrm{H}$ I have different velocities and temperatures. The same problem was found in Paper III for the single-component fits to the $\alpha$ Cen $A$ and $B$ spectra. The $\mathrm{H} I$ lines of both $\epsilon$ Ind and $\lambda$ And appear to be blueshifted relative to the D I lines by about 3 $\mathrm{km} \mathrm{s}^{-1}$. Temperatures for $\mathrm{H}$ I and $\mathrm{D}_{\mathrm{I}}$ can be computed from the $b$ values in Table 2 by using the equation $b^{2}=0.0165 T / A+\xi^{2}$, where $A$ is the atomic weight (1 for
$\mathrm{H} \mathrm{I}, 2$ for D I) and $\xi$ is the nonthermal velocity. We cannot measure $\xi$ as we did in Papers I- III without observations of the $\mathrm{Mg}$ II and $\mathrm{Fe}$ II absorption lines, so throughout this paper we will simply use the value measured in Paper III, $\xi=1.2 \mathrm{~km} \mathrm{~s}^{-1}$, which is also consistent with the results of Papers I and II. The exact value used is not too important since Papers I-III have conclusively shown that LISM H I and $D_{I}$ lines are dominated by thermal rather than nonthermal broadening. For $\epsilon$ Ind, the $\mathrm{H}$ i temperature, 8620 $\mathrm{K}$, is significantly hotter than the D I temperature, $6980 \mathrm{~K}$. A similar discrepancy is seen for $\lambda$ And, where the $\mathrm{H} \mathrm{I}$ and D I temperatures are 13,040 and $10,000 \mathrm{~K}$, respectively.

For $\lambda$ And, we computed another single-component fit in which we forced the velocities and temperatures of $D_{1}$ and $\mathrm{H}$ I to be consistent. The resulting fit was very poor, so we used the residuals of that fit to dramatically alter the assumed stellar Ly $\alpha$ profile, and then we fit the data again. The result is shown in Figure 5. In trying to force consistency between $\mathrm{D}_{\mathrm{I}}$ and $\mathrm{H}$ I, we were forced to make the stellar profile (Fig. 5, solid line) very asymmetric with a very strong red peak. This is not a very reasonable profile, but in any case, the fit resulting from the use of this profile still is not very good. The flux excess at $1215.3 \AA$ seen in Figure $5 b$ indicates that the $\mathrm{D} I$ line velocity is more redshifted than the fit suggests, and the flux deficit near $1215.45 \AA$ indicates that the $\mathrm{H}$ I line velocity is actually more blueshifted than the fit suggests. Trying once more to use the residuals of the fit to alter the stellar profile and improve the fit, we derive the profile shown as a dotted line in Figure $5 a$. This profile

TABLE 2

Parameters for the Single-Component Fits

\begin{tabular}{cccrcccc}
\hline \hline Star & Ion & Line & \multicolumn{1}{c}{$\begin{array}{c}\text { Velocity } \\
\left(\mathrm{km} \mathrm{s}^{-1}\right)\end{array}$} & \multicolumn{1}{c}{$\begin{array}{c}b \\
\left(\mathrm{~km} \mathrm{~s}^{-1}\right)\end{array}$} & $\log N$ & \multicolumn{1}{c}{$\tau$} & $\chi_{v}^{2}$ \\
\hline$\epsilon$ Ind $\ldots . .$. & H I & 1215.670 & $-12.6 \pm 0.1$ & $11.98 \pm 0.09$ & $18.292 \pm 0.003$ & 123,000 & 1.361 \\
& D I & 1215.339 & $-9.9 \pm 0.1$ & $7.68 \pm 0.19$ & $13.127 \pm 0.011$ & 1.31 & 1.361 \\
$\lambda$ And $\ldots . .$. & H I & 1215.670 & $+3.3 \pm 0.1$ & $14.71 \pm 0.16$ & $18.645 \pm 0.002$ & 227.000 & 1.095 \\
& D I & 1215.339 & $+6.6 \pm 0.1$ & $9.16 \pm 0.18$ & $13.658 \pm 0.013$ & 3.74 & 1.095 \\
\hline
\end{tabular}




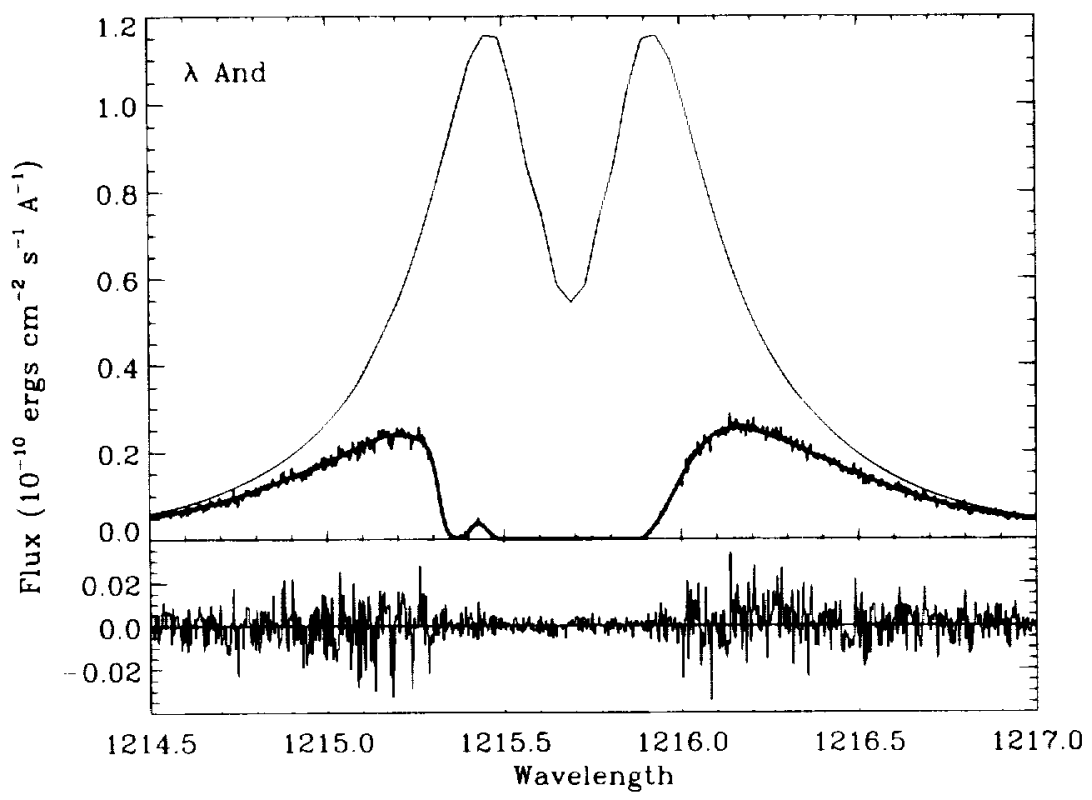

Fig. 4.-Our best single-component fit to the $\mathrm{H}_{\mathrm{I}}$ and $\mathrm{D}_{\mathrm{I}}$ absorption lines of $\lambda$ And, with the residuals displayed below the fit. The data are shown in histogram form. The thin solid line is the assumed stellar Ly $\alpha$ profile, and the thick solid line is the fit. The parameters for the fit are given in Table 2.

is even less plausible, however, because of the rapid flux changes between $1214.3 \AA$ and $1215.5 \AA$. We conclude that the only way to force consistency between $\mathrm{D} I$ and $\mathrm{H} I$ is to add a second $\mathrm{H} \mathrm{I}$ absorption component.

For $\epsilon$ Ind, we mention yet another problem with the fit in Figure 3. The upper part of the assumed stellar profile is

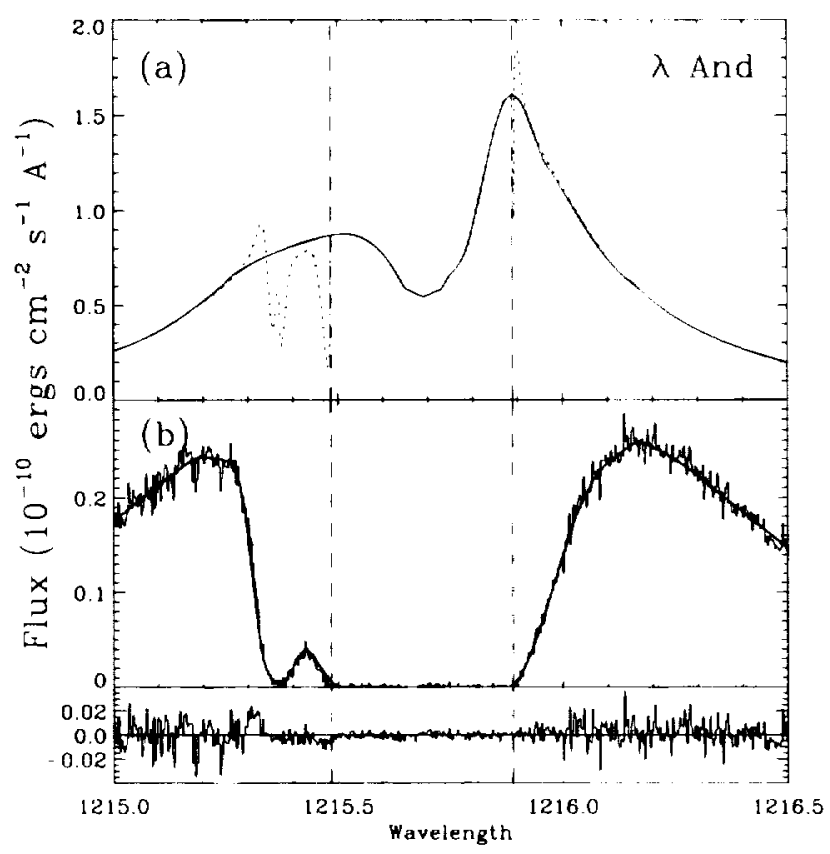

FIG. 5.- Our best single-component fit to the $\mathrm{H} I$ and $\mathrm{D} I$ absorption lines of $\lambda$ And in which $\mathrm{H} I$ and $D \mathrm{I}$ are forced to have the same velocity and temperature. The vertical dashed lines mark the boundaries of the saturated core of the $\mathrm{H} \mathrm{I}$ absorption. Between these lines, the shape of the assumed stellar profile has no effect on the quality of the fit. The assumed profile, shown as a solid line in $(a)$, has been dramatically altered from that assumed in Fig. 4 in order to maximize the quality of the fit, but $(b)$ reveals that there are still significant discrepancies between the fit and the data. When the residuals of the fit are used to alter the stellar profile in an attempt to improve the fit, the profile shown as a dotted line in (a) results. This is not a reasonable profile, suggesting that $\mathrm{H}$ I and $\mathrm{D}$ I cannot be forced to be consistent in a single-component fit. redshifted by $5-10 \mathrm{~km} \mathrm{~s}^{-1}$ from the stellar radial velocity of $-38.9 \pm 0.3 \mathrm{~km} \mathrm{~s}^{-1}$ (Buscombe \& Kennedy 1968). The far wings of $\epsilon$ Indi's Ly $\alpha$ line, away from the effects of the interstellar absorption, do not show this redshift, and there is no evidence that the Ly $\alpha$ lines of inactive main-sequence stars like $\epsilon$ Ind should have significant redshifts. For example, the Lyman- $\alpha$ lines of $\alpha$ Cen A, $\alpha$ Cen B, and Procyon appear to be well centered on the stellar radial velocity (see Papers II, III; Wood et al. 1996). All of the single-component fits to the $\epsilon$ Ind data that we have tried (assuming different stellar profiles, forcing $\mathrm{D}_{\mathrm{I}}$ and $\mathrm{H}$ I to be consistent, etc.) suggested $\log N_{\mathrm{HI}} \approx 18.3$. We will now show that this value cannot be correct because this value is inextricably linked with unreasonably redshifted stellar profiles.

Primarily because of the large radial velocity of $\epsilon$ Ind $\left(v=-38.9 \pm 0.3 \mathrm{~km} \mathrm{~s}^{-1}\right.$; Buscombe \& Kennedy 1968), the interstellar $\mathrm{H}_{\mathrm{I}}$ absorption is redshifted by about $30 \mathrm{~km} \mathrm{~s}^{-1}$ from the center of the stellar emission line, and therefore, there is more absorption in the red wing of the line than in the blue wing. Thus, the wings of the observed Ly $\alpha$ profile are blueshifted from the expected velocity of $-38.9 \mathrm{~km} \mathrm{~s}^{-1}$. By assuming different values for $N_{\mathrm{H}}$, we can reconstruct the wings of the intrinsic stellar Ly $\alpha$ profile. When the correct value for $\log N_{\mathrm{H} I}$ is assumed, the result should be a profile centered at $-38.9 \mathrm{~km} \mathrm{~s}^{-1}$. This process is illustrated in Figures 6 and 7. The numbers in Figure 6 are assumed values for $\log N_{\mathrm{HI}}$, and the dotted lines associated with these numbers are the wings of the intrinsic stellar Ly $\alpha$ line computed on the basis of these values by multiplying the observed flux by $\exp \left(+\tau_{\lambda}\right)$, where $\tau_{2}$ is the optical depth of the damping wing of the interstellar hydrogen absorption line. We assume that the interstellar $\mathrm{H}$ I absorption is centered on the D I velocity listed in Table 2 . These computations rely on completely smooth estimates of the observed line wings (Fig. 6, thick solid lines) obtained using polynomial fits to the data.

In order to determine which value of $\log N_{\mathrm{H}}$ leads to an intrinsic stellar profile whose wings are best centered on the stellar radial velocity, we compute bisectors for the com- 


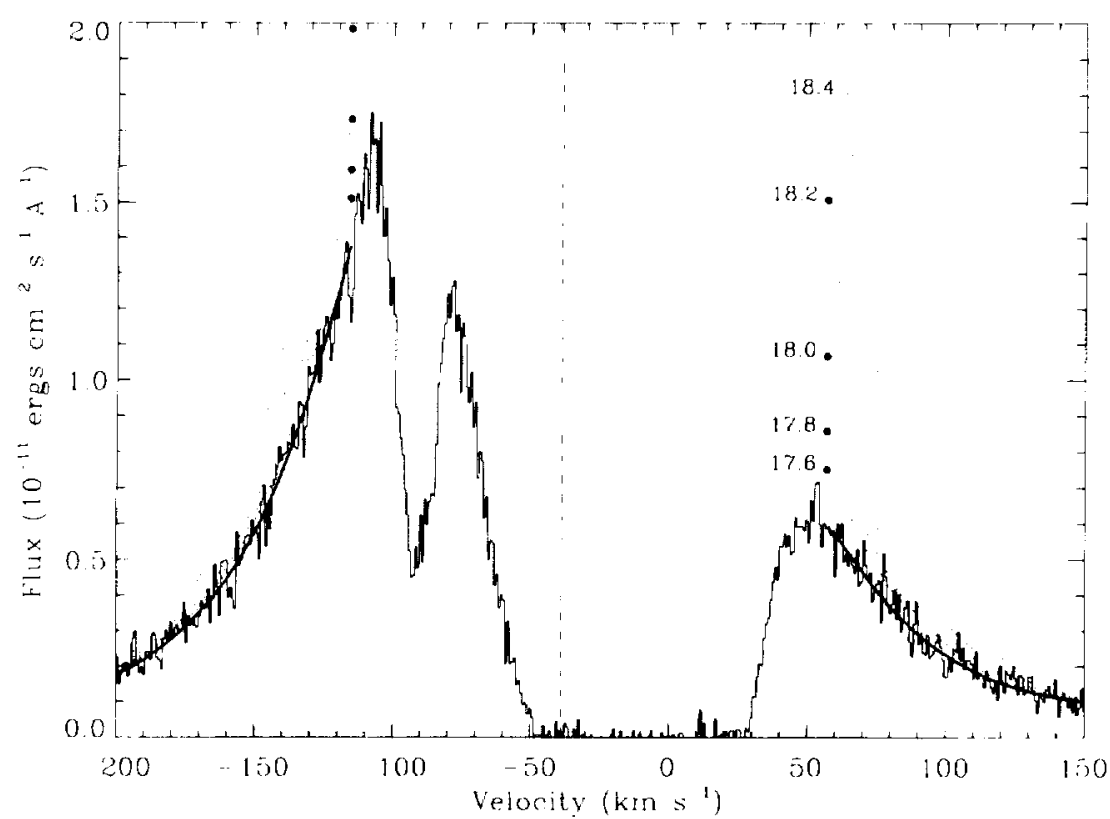

FIG. 6.-Reconstructions of the wings of the Ly $\alpha$ profile of $\epsilon$ Ind, assuming different values for the interstellar $\mathrm{H}_{\mathrm{I}}$ column density, log $\mathrm{N}_{\mathrm{H} \mathrm{I}}$. The data are displayed in histogram form. The thick solid lines are polynomial fits to the wings of the observed Ly $\alpha$ profile. The wings of the intrinsic stellar Ly $\alpha$ profile (dotted lines) are computed for five different values for $\log \mathrm{N}_{\mathrm{HI}}$ (the numbers in the figure), using the polynomial fits as smoothed representations of the data. The correct value of $\log \mathrm{N}_{\mathrm{H}}$ should produce a stellar profile centered on the stellar radial velocity of $-38.9 \mathrm{~km} \mathrm{~s}^{-1}$, which is marked by the dashed line.

puted profiles. Figure 7 shows the bisectors of the intrinsic stellar Ly $\alpha$ line computed for many different values of $\log N_{\mathrm{HI}}$ (the numbers in Fig. 7). The radial velocity of the star is indicated by the vertical dashed line. For $\log N_{\mathrm{H} 1}=$ 17.6-17.8, the bisector is blueshifted relative to the expected velocity by $2-3 \mathrm{~km} \mathrm{~s}^{-1}$, whereas for $\log \mathrm{N}_{\mathrm{HI}}=18.2-18.4$, the bisector is redshifted relative to the expected velocity by 2-6 $\mathrm{km} \mathrm{s}^{-1}$. The value of $\log \mathrm{N}_{\mathrm{H} \text { I }}$ that produces a stellar Ly $\alpha$ profile that is best centered on the radial velocity of the star is $\log N_{H I}=18.03$. The bisector for this profile is shown as a solid line in Figure 7.
Ideally, this bisector should be a vertical line exactly at the expected velocity. The obvious deviations from this ideal are probably due to the uncertainties involved in estimating the shape of the observed profile using polynomial fits and the uncertainties involved in the computation of the bisectors. Since this analysis is dependent on an accurate stellar radial velocity and an accurate wavelength calibration, uncertainties in both of these things are potential sources of error. After consideration of all the sources of uncertainty mentioned above, we report a value and error for the hydrogen column density of $\log \mathrm{N}_{\mathrm{HI}}=18.0 \pm 0.1$.

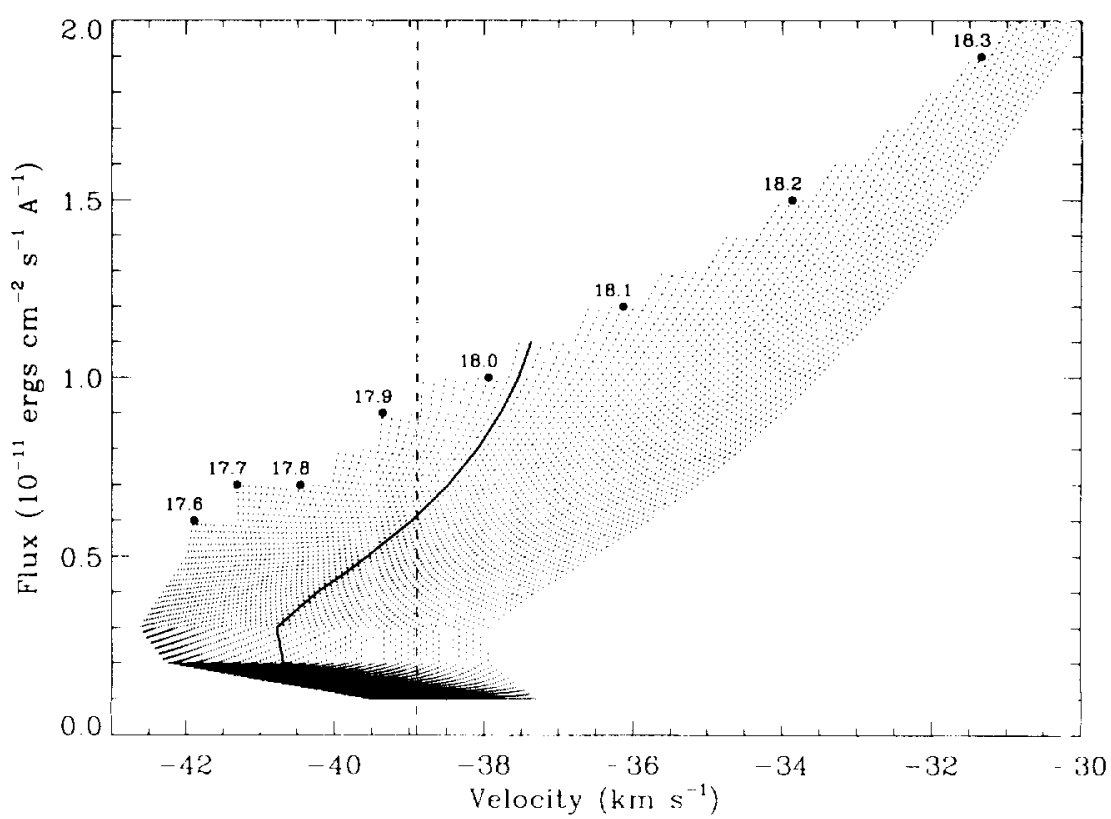

Fig. 7.-Dotted lines are bisectors of the intrinsic Ly $\alpha$ profile of $\epsilon$ Ind computed (as illustrated in Fig. 6) for many different values for log $N_{H}$ (the numbers in the figure). The bisector that best agrees with the stellar radial velocity of $-38.9 \mathrm{~km} \mathrm{~s}^{-1}$ (dashed line) is shown as a solid line. This bisector was computed assuming $\log \mathrm{N}_{\mathrm{HI}}=18.03$. 


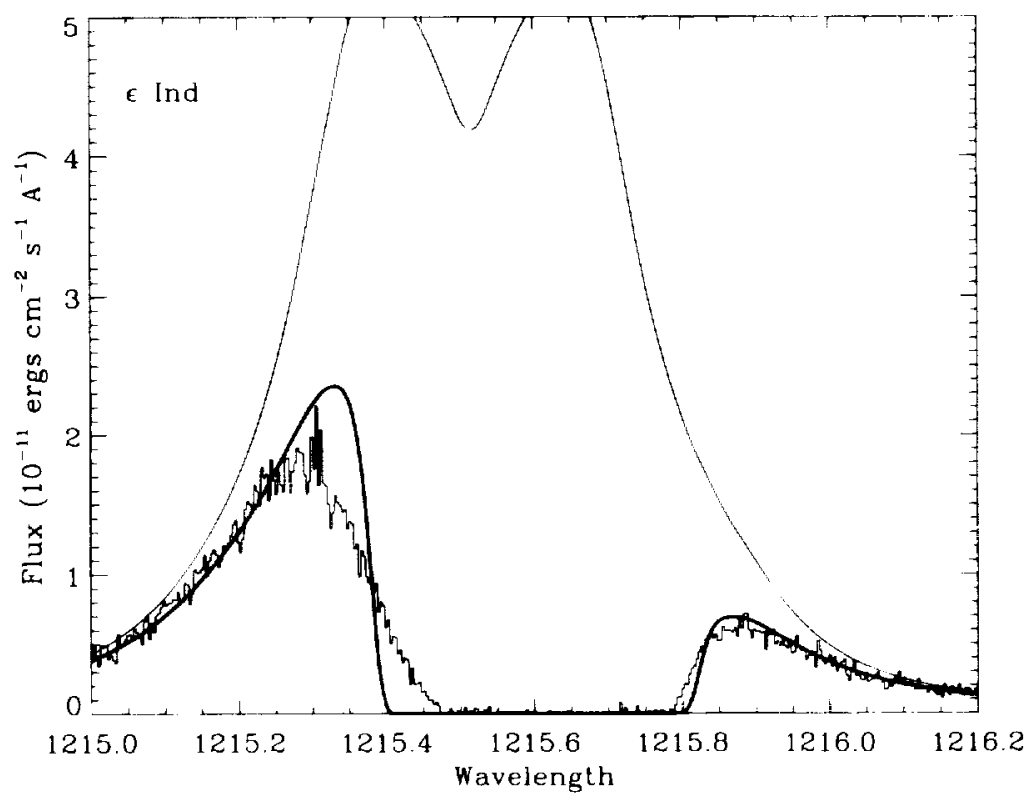

Frg. 8. - Single-component fit to the $\mathrm{H} \mathrm{I}$ absorption line of $\epsilon \mathrm{Ind}$, in which the $\mathrm{D}$ I line has been removed using the $\mathrm{D}$ I parameters listed in Table 2 . The assumed stellar Ly $\alpha$ profile was created using the $\log N_{H I}=18.03$ value measured from Fig. 7. In this fit, we have in fact forced log $N_{H I}=18.0$. The resulting fit is very poor, demonstrating that a single-component fit cannot fit the data if $\log \mathrm{N}_{\mathrm{H} I}$ is in fact $18.0 \pm 0.1$.

Note that $\log \mathrm{N}_{\mathrm{H} I}=18.3$, the value suggested by the singlecomponent fits, is well outside these error bars.

Now that we have a measurement of $\log \mathrm{N}_{\mathrm{H} \text { I }}$, we can create a stellar profile designed with this value in mind. We start with $\mathrm{Ly} \alpha$ line wings computed like those in Figure 6 assuming $\log N_{\mathrm{H} I}=18.0$. We then model the center of the line profile with the $\alpha$ Cen B profile, which was also used in deriving the profile in Figure 3. Using this profile, in Figure 8 we perform a single-component fit to the $\mathrm{H}$ I line, requiring $\log \mathrm{N}_{\mathrm{H}}$ to be 18.0 . For this fit, we removed the $\mathrm{D}$ I line from the data using the $\mathrm{D}$ I parameters in Table 2 in order to focus attention on the $\mathrm{H}_{\mathrm{I}}$ absorption, the idea being to show that $\log \mathrm{N}_{\mathrm{H} \text { I }}=18.0$ cannot lead to an acceptable single-component fit regardless of whether or not the parameters for $\mathrm{D} I$ and $\mathrm{H} I$ are consistent. This is illustrated by the very poor quality of the fit in Figure 8 . Absorption lines with $\log \mathrm{N}_{\mathrm{HI}}=18.0$ have sides that are simply too steep to match the $\epsilon$ Ind data. Thus, a single-component fit cannot work for the $\epsilon$ Ind line of sight.

\section{TWO-COMPONENT FITS TO THE DATA}

\subsection{Analysis of the $\epsilon$ Ind Data}

We have concluded that a second $\mathrm{H}$ I absorption component must be present for both $\epsilon$ Ind and $\lambda$ And to fit the data adequately and to explain the apparent discrepancies between $\mathrm{H}_{\mathrm{I}}$ and $\mathrm{D}_{\mathrm{I}}$. Based on the results of Paper III, the most likely sources for the additional absorption are solar and/or stellar hydrogen walls.

For $\epsilon$ Ind, initial two-component fits were performed, and the stellar profile was then altered slightly to improve the quality of the fits. In all of our two-component fits, we force the $D_{\mathrm{I}}$ and $\mathrm{H}$ I lines for each component to have the same velocities and temperatures for both components. We also force the two components to have the same $\mathrm{D} / \mathrm{H}$ ratio, although the D I column density of the second component is too small to produce any noticeable absorption.

The final result is shown in Figure 9, and the fit parameters are given in Table 3. The dotted line in Figure 9 represents the absorption only from the ISM. The second absorption component (dashed line) is blueshifted relative the ISM component, explaining why the $\mathrm{H}$ I line was blueshifted relative to $D_{I}$ in the single-component fits. The two-component fit to the $\epsilon$ Ind data is clearly a better match to the data than the single-component fit. The singlecomponent model did not fit the data well, mostly because the $\mathrm{H}$ I absorption line appears to have an asymmetry in which the red side of the line is somewhat steeper than the blue side (see Fig. 3). This asymmetry is nicely explained by the two-component fit (see Fig. 9).

TABLE 3

Parameters for the Two-Component Frts

\begin{tabular}{ccccccc}
\hline \hline Star & $\begin{array}{c}\text { Velocity } \\
\left(\mathrm{km} \mathrm{s}^{-1}\right)\end{array}$ & $\begin{array}{c}b_{\mathrm{H} \mathrm{I}} \\
\left(\mathrm{km} \mathrm{s}^{-1}\right)\end{array}$ & $\log \mathrm{N}_{\mathrm{HI}}$ & $\begin{array}{c}\mathrm{D} / \mathrm{H} \\
\left(10^{-5}\right)\end{array}$ & $\tau$ & $\chi_{\mathrm{v}}^{2}$ \\
\hline$\epsilon$ Ind $\ldots \ldots .$. & $-9.4 \pm 0.1$ & $12.15 \pm 0.10$ & $17.949 \pm 0.019$ & $1.74 \pm 0.12$ & 55,300 & 1.117 \\
& $-27.8 \pm 1.5$ & $41.39 \pm 1.20$ & $14.244 \pm 0.031$ & $(1.74 \pm 0.12)$ & 3.21 & 1.117 \\
$\lambda$ And $\ldots . .$. & $+6.5 \pm 0.1$ & $13.87 \pm 0.18$ & $18.455 \pm 0.002$ & $1.72 \pm 0.06$ & 155,000 & 1.149 \\
& $-2.0 \pm 1.1$ & $31.89 \pm 1.66$ & $14.722 \pm 0.120$ & $(1.72 \pm 0.06)$ & 12.5 & 1.149 \\
\hline
\end{tabular}




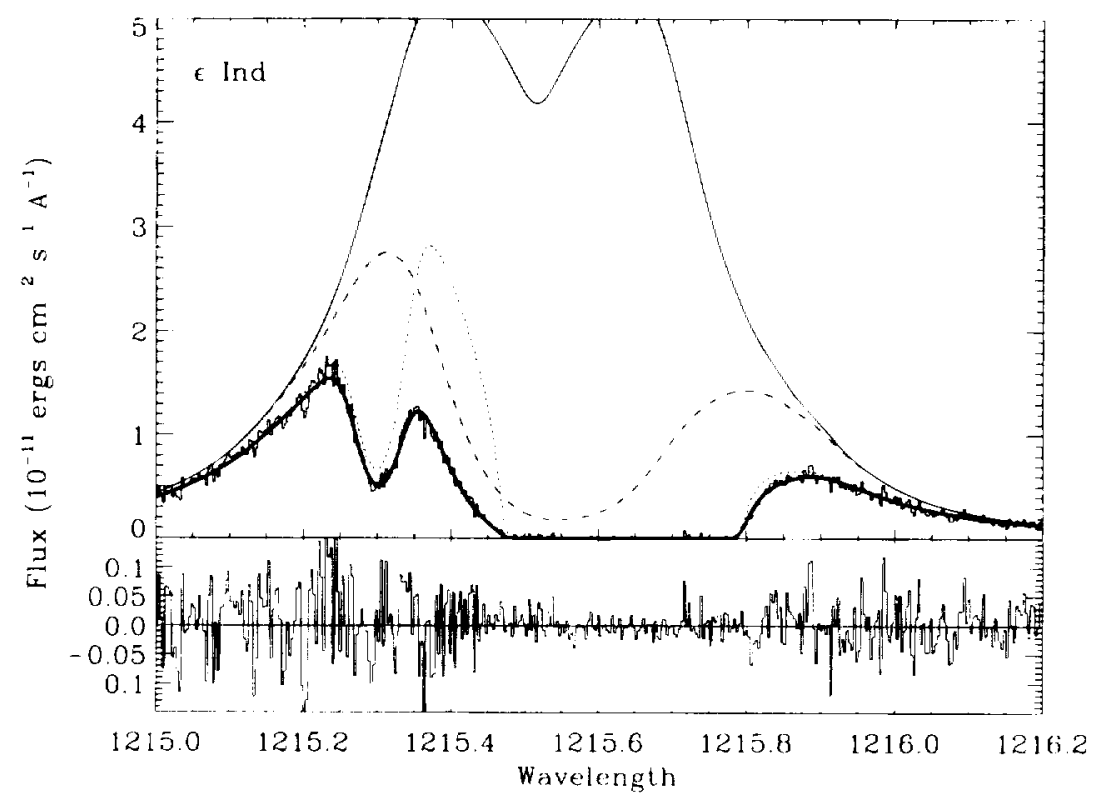

FIG. 9.-Our best two-component fit to the $\mathrm{H} \mathrm{I}$ and $\mathrm{D} \mathrm{I}$ absorption lines of $\epsilon$ Ind, with the residuals displayed below the fit. The data are shown in histogram form. The thin solid line is the assumed stellar Ly $\alpha$ profile. The dotted line is the component representing absorption from the ISM, the dashed line is the component representing absorption from a hydrogen wall surrounding $\epsilon$ Ind (see $\$ 4.1$ ), and the thick solid line is the combination of the two absorption components. The parameters for the fit are listed in Table 3.

Because hydrogen-wall material around a star should be decelarated by its interaction with the stellar wind, the velocity of this material should generally lie between the stellar velocity and that of the ISM. Thus, for the $\epsilon$ Ind line of sight, the solar hydrogen wall should have a velocity between 0 and $-9.2 \mathrm{~km} \mathrm{~s}^{-1}$. However, any material at a velocity in this range should cause the $\mathrm{H} \mathrm{I}$ absorption line to be redshifted relative to the $D_{I}$ line rather than blueshifted as observed. We measure the second absorption component of the $\mathrm{H} \mathrm{I}$ absorption line to be at $-27.8 \mathrm{~km} \mathrm{~s}^{-1}$ (see Table 3 ), inconsistent with the solar hydrogen wall. Furthermore, the temperature inferred for this component from the Doppler parameter $\left(h_{\mathrm{HI}}=41.39 \mathrm{~km} \mathrm{~s}^{-1}\right)$ is $104,000 \mathrm{~K}$, which is much hotter than the temperature measured for the solar hydrogen wall in Paper III. Since the velocity and temperature of the second component are inconsistent with the solar hydrogen wall, we instead attribute this component to an analogous hydrogen wall surrounding the star. The velocity of the second component is certainly consistent with this interpretation as is the temperature for reasons that we will present in $\$ 6$.

Finally, we discuss the effects that multiple ISM components might have on our analysis. Unfortunately, we do not have observations of narrower ISM absorption lines of heavier atomic species, such as $\mathrm{Mg}$ II and Fe II, that would show whether multiple components exist. We believe, however, that even if there are multiple velocity components, a very hot component like the second component in Figure 9 must still be present to fit the observed absorption profile. No combination of typical $T \approx 7000 \mathrm{~K}$ LISM absorption components can explain the curved appearance of the blue side of the $\mathrm{H}$ I absorption line, and no alteration of the assumed stellar profile can explain this either without introducing unreasonable fine structure into the profile (see $\S 3$ ). However, a very hot $T \approx 100,000 \mathrm{~K}$ absorption component with a low column density like that shown in Figure 9 fits the data quite nicely.

\subsection{Analysis of the $\lambda$ And Data}

The "bisector method" for measuring $N_{\mathrm{H}}$ could not have been used for either $\alpha$ Cen of Capella in Papers I-III, because for these lines of sight the velocity separations between the stellar emission and the interstellar absorption are small. For the same reason, we cannot use the bisector method in the analysis of the $\lambda$ And data. Therefore, for our inital two-component fit to the Ly $\alpha$ profile of $\lambda$ And, we simply assume the same stellar profile that was used for the single-component fit in Figure 4. As we did for the twocomponent fits in $\$ 4.1$, we force the velocities and temperatures of the $\mathrm{H}$ I and $\mathrm{D}_{\mathrm{I}}$ lines to be the same, and we require the $\mathrm{D} / \mathrm{H}$ ratio to be the same for both components. Figure 10 shows the resulting fit. The dotted line shows the absorption only from the ISM component, and the dashed line represents the absorption from the second component. We would like to associate this second component with either the solar hydrogen wall or a stellar one. To see if this is possible, we first determine if the vclocity of the second component, $-31 \mathrm{~km} \mathrm{~s}^{-1}$, is consistent with such an interpretation.

Our observations of $\lambda$ And were made at orbital phase $\phi=0.91$, when the G8 IV III star has a velocity of +3.3 $\mathrm{km} \mathrm{s}^{-1}$ (Batten et al. 1978). However, if the solar analogy is any guide, it should take at least a year for stellar wind material to reach the hydrogen wall of $\lambda$ And, if it has one, and since the orbital period of $\lambda$. And is only 20.5 days, we doubt that the hydrogen wall is affected by orbital motions. Thus, the center-of-mass velocity of the $\lambda$ And system, +6.8 $\mathrm{km} \mathrm{s}^{-1}$, is the relevant velocity for estimating the velocity expected for a stellar hydrogen wall. The velocities of the solar and/or stellar hydrogen witls should not be too different from the velocity of the interstellar absorption $(+6 \mathrm{~km}$ $\left.\mathrm{s}^{-1}\right)$, because both the Sun's velocity $\left(0 \mathrm{~km} \mathrm{~s}^{-1}\right)$ and the center-of-mass velocity of the $i$ And binary system $(+6.8$ $\mathrm{km} \mathrm{s}^{-1}$ ) are not that different from this value. While we 


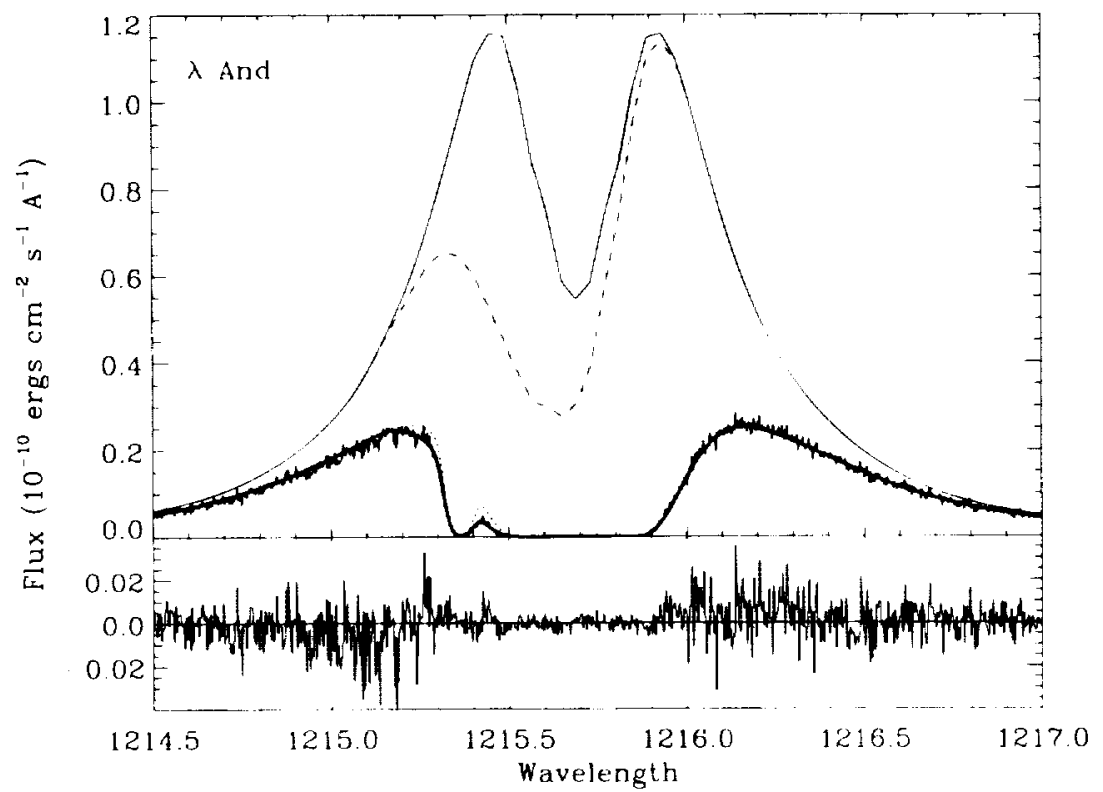

Fig. 10.-A two-component fit to the $\mathrm{H}_{\mathrm{I}}$ and $\mathrm{D}$ I absorption lines of $\lambda$ And, assuming the same stellar Ly $\alpha$ profile (thin solid line) used in the single-component fit in Fig. 4. The data are shown in histogram form. The dotted line is the component representing absorption from the ISM, the dashed line is the second component for which we have no interpretation (see $\$ 4.2$ ), and the thick solid line is the combination of the two components.

expect the second component to be blueshifted relative to the interstellar absorption component at $+6 \mathrm{~km} \mathrm{~s}^{-1}$, in order to explain why $\mathrm{H} I$ is blueshifted relative to $D_{I}$ (see Table 2), the blueshift of the second component in Figure 10 $\left(-31 \mathrm{~km} \mathrm{~s}^{-1}\right)$ is clearly too large to be explained by a solar or stellar hydrogen wall.

We tried another two-component fit in which we forced the velocity of the second component to be $0 \mathrm{~km} \mathrm{~s}^{-1}$, but the quality of that fit was not particularly good. We then experimented with different intrinsic stellar profiles to see whether we could find one that would lead to a twocomponent fit with a second component that we could attribute to a hydrogen wall. We found that broader stellar profiles designed to produce fits with smaller values for $N_{\mathrm{H} \mathrm{I}}$ yielded promising results. Figure 11 shows the best of these fits. The parameters of this fit are given in Table 3. The

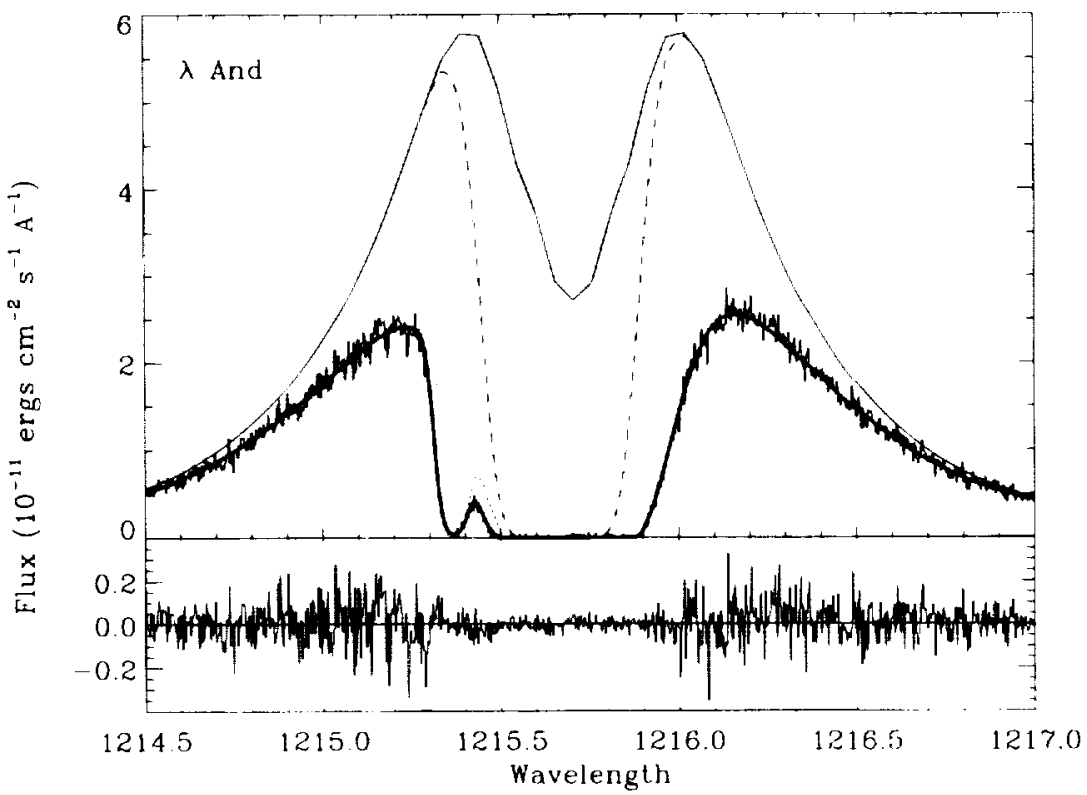

FIG. 11. - Our best two-component fit to the $\mathrm{H}$ I and $\mathrm{D}$ I absorption lines of $\lambda$ And, with the residuals displayed below the fit. The data are shown in histogram form. The thin solid line is the assumed stellar Ly $\alpha$ profile. The dotted line is the component representing absorption from the ISM, the dashed line is the component representing absorption from a hydrogen wall surrounding $\lambda$ And (see $\$ 4.2$ ), and the thick solid line is the combination of the two components. The parameters for the fit are given in Table 3 . We consider this fit to be superior to the two-component fit in Fig. 10 because for this fit we can interpret the second absorption component as being due to a stellar hydrogen wall, which is not the case for the fit in Fig. 10. 
velocity of the second component is $-2 \mathrm{~km} \mathrm{~s}^{-1}$, which is close enough to the solar rest frame to be consistent with the solar hydrogen wall. However, the Doppler parameter of this component $\left(b=31.89 \mathrm{~km} \mathrm{~s}^{-1}\right)$ suggests a temperature of $62,000 \mathrm{~K}$, which is much hotter than the 29,000 $\mathrm{K}$ temperature measured for the solar hydrogen wall in Paper III. In fact, at a temperature of $29,000 \mathrm{~K}$, the solar hydrogen wall does not produce an absorption component broad enough to be detected, given the very broad $\mathrm{H}$ I absorption feature we observe in our $\lambda$ And data. Thus, we believe that the second component in Figure 11 must be due to a stellar hydrogen wall. The high temperature of the stellar wall component and the $8 \mathrm{~km} \mathrm{~s}^{-1}$ blueshift of this absorption with respect to the interstellar absorption and $\lambda$ And rest frames have reasonable explanations that will be discussed in $\S 6$.

For $\epsilon$ Ind, we argued that the hot second component is necessary to fit the data regardless of whether or not there are multiple ISM components along the line of sight. However, we cannot be sure that this is the case for $\lambda$ And. In contrast to $\epsilon$ Ind, the $H$ I line of $\lambda$ And is nicely fitted by a single ISM component, the only problem being that the parameters of $\mathrm{H}$ I and D I are inconsistent. It is not immediately apparent why the second absorption component for $\lambda$ And cannot be an ISM component with a much higher column density and much lower temperature than the second component in Figure 11. We tried to force our twocomponent fits to produce such a component. The resulting fits were not as good as that shown in Figure 11, but they were not too bad, and the discrepancies between the fits and the data were far more subtle than the discrepancies seen for the $\epsilon$ Ind fits in Figures 3 and 8, for example. Nevertheless, the fit shown in Figure 9 is our best two-component fit for $\lambda$ And, and the parameters for the hot component do conform nicely to what we expect for a hydrogen wall around $\lambda$ And (see $\S 6$ ), but these results must be considered tentative. If future observations reveal multiple ISM components for this line of sight, these data should be reanalyzed with this in mind.

\subsection{Estimating Systematic Errors}

The errors cited in Table 3 are only the random errors of the fitting process and do not include estimates of systematic errors, such as those that result from uncertainties in the adopted stellar $\mathrm{Ly} \alpha$ profiles. In an attempt to assess these systematic errors, we experimented with a large number of possible stellar profiles to determine which profiles produce two-component fits that match the data well but have significantly different parameters than the fits shown in Figures 9 and 11. Deciding whether or not a fit matches the data well enough to be considered acceptable is somewhat subjective, but this is a necessary step in estimat- ing the uncertainties in the assumed stellar profiles, which are the dominant sources of systematic error in the analysis.

For $\epsilon$ Ind, the interstellar $\mathrm{H}$ I column density derived from the bisector analysis $\left(\log \mathrm{N}_{\mathrm{HI}}=18.0 \pm 0.1\right)$ provides an important guideline that can be used to separate the acceptable fits from the unacceptable. For $\lambda$ And, we required that the second component of the two-component fit be at a velocity consistent with a stellar hydrogen wall. As Figure 10 suggests, not all assumed stellar profiles resulted in fits that met this criterion. Although absorption from a hydrogen wall around $\alpha$ Cen was not really detected in Paper III, the possibility that this absorption is present and affects the data was considered in estimating errors for the fit parameters. Similarly, we experimented with threecomponent fits to the Ly $\alpha$ line of $\epsilon$ Ind to see how the addition of a component representing the solar hydrogen wall could change the derived parameters of the other two components. The results from Paper III were used to constrain the parameters of the solar hydrogen-wall component. Since the temperature of the solar hydrogen wall measured in Paper III implies an absorption feature too narrow to have affected the very broad $\mathrm{H}$ I absorption line seen in the $\lambda$ And data, we did not experiment with threecomponent fits to the Ly $\alpha$ line of $\lambda$ And.

In Table 4, we show the results of these experiments. This table summarizes the results of the two-component fit analysis, and the uncertainties in the fit parameters include our estimates of the systematic errors.

\section{THE PROPERTIES OF THE INTERSTELLAR MEDIUM}

The $V_{\mathrm{G}}$ and $V_{\mathrm{AG}}$ flow vectors of Lallement \& Bertin (1992) predict LISM velocities for the $\epsilon$ Ind line of sight of -8.5 and $-8.9 \mathrm{~km} \mathrm{~s}^{-1}$, respectively. These two velocities are too close for us to determine whether $\epsilon$ Ind is in the $G$ cloud or in the AG cloud. Our measured velocity of $-9.2 \pm 1.0 \mathrm{~km}$ $\mathrm{s}^{-1}$ (see Table 4) is consistent with both velocities. Because $\epsilon$ Ind is located in the general direction of the Galactic center, it would seem more likely that $\epsilon$ Ind is in the $G$ cloud. However, the LISM temperature that we have measured $(8500 \pm 500 \mathrm{~K})$ is much hotter than the temperature $(5400 \pm 500 \mathrm{~K})$ measured for the $\alpha$ Cen line of sight, which is definitely in the $G$ cloud (Paper III). The $\epsilon$ Ind temperature is also somewhat hotter than the temperatures of $7000 \pm 900 \mathrm{~K}$ and $6900 \pm 380 \mathrm{~K}$ measured for the Capella and Procyon lines of sight, respectively, which are in the AG cloud (Paper II). Observations of the interstellar Mg II and Fe II lines are needed to verify that the Doppler parameter we have measured is indeed an accurate measure of the LISM temperature and has not been artificially broadened by the presence of more than one velocity component.

The $\mathrm{D} / \mathrm{H}$ ratio that we have measured for the $\epsilon$ Ind line of sight, $(1.6 \pm 0.4) \times 10^{-5}$, is in excellent agreement with the

TABLE 4

SUmmary of QuaNTITative Results (Systematic Errors Included)

\begin{tabular}{ccccccc}
\hline \hline Star & $\begin{array}{c}\text { Velocity } \\
\left(\mathrm{km} \mathrm{s}^{-1}\right)\end{array}$ & $\begin{array}{c}b_{\mathrm{HI}} \\
\left(\mathrm{km} \mathrm{s}^{-1}\right)\end{array}$ & $\begin{array}{c}T \\
\left(10^{3} \mathrm{~K}\right)\end{array}$ & $\log \mathrm{N}_{\mathrm{HI}}$ & $\begin{array}{c}\mathrm{D} / \mathrm{H} \\
\left(10^{-5}\right)\end{array}$ & Source of Absorption \\
\hline$\epsilon$ Ind $\ldots . . .$. & $-9.2 \pm 1.0$ & $11.9 \pm 0.3$ & $8.5 \pm 0.5$ & $18.0 \pm 0.1$ & $1.6 \pm 0.4$ & ISM \\
& $-27 \pm 6$ & $41 \pm 4$ & $100 \pm 20$ & $14.2 \pm 0.2$ & $(1.6 \pm 0.4)$ & Stellar H I wall \\
$\lambda$ And ...... & $+6.5 \pm 1.0$ & $13.8 \pm 0.3$ & $11.5 \pm 0.5$ & $18.45 \pm 0.15$ & $1.7 \pm 0.5$ & ISM \\
& $-2 \pm 3$ & $32 \pm 5$ & $62 \pm 18$ & $14.8 \pm 0.2$ & $(1.8 \pm 0.5)$ & Stellar H I wall \\
\hline
\end{tabular}


values measured in Papers I-III. This agreement is very encouraging, since the $\mathrm{H}$ I column density was measured using an entirely different technique than in previous analyses namely the bisector method. The $\mathrm{H}$ I column density toward $\epsilon$ Ind, $\log \mathrm{N}_{\mathrm{H} I}=18.0 \pm 0.1$, implies an average number density of $n_{\mathrm{HI}}=0.094 \pm 0.022 \mathrm{~cm}^{-3}$. This density is consistent with the densities measured toward Procyon and $\alpha$ Cen, which are both less than $4 \mathrm{pc}$ from the Sun, as is $\epsilon$ Ind. Note that the bisector analysis and our best two-component fit resulted in an $\mathrm{H}_{1}$ column density a factor of 2 different from that computed in the singlecomponent fit $\left(\log \mathrm{N}_{\mathrm{HI}}=18.292\right)$. This illustrates that absorption components that are present but left out of the analysis can result in inaccurate column densities, regardless of the quality of the data.

The flow velocities predicted for the $\lambda$ And line of sight by the $V_{\mathrm{G}}$ and $V_{\mathrm{AG}}$ vectors are +9.8 and $+7.6 \mathrm{~km} \mathrm{~s}{ }^{1}$, respectively. We expect the $\lambda$ And line of sight to be in the AG cloud on the basis of its Galactic coordinates, and the observed velocity of the ISM absorption, $+6.5 \pm 1.0 \mathrm{~km}$ $\mathrm{s}^{-1}$, is indeed close to the expected velocity. However, the temperature of $11.500 \pm 500 \mathrm{~K}$ that we have measured is substantially higher than the temperature of $7000 \mathrm{~K}$ measured for the Capella and Procyon lines of sight, which are both predominantly in the AG cloud. This temperature is also higher than the $8500 \mathrm{~K}$ value that we have estimated from the $\epsilon$ Ind data. For $\epsilon$ Ind, we expressed concern that unresolved ISM components might be broadening the D I and $\mathrm{H} \mathrm{I}$ absorption lines. This concern is even greater for $\lambda$ And, because the discrepancy with previous measurements is larger and because the longer $(23 \mathrm{pc})$ line of sight to $\lambda$ And makes it far more likely that multiple components are present. If future observations should confirm that multiple components are present toward $\lambda$ And, our spectrum of the Lyo line should be reanalyzed with this in mind, as we previously emphasized in $\$ 4.2$. It is of course possible that the average ISM temperature toward $\lambda$ And really is hotter than for the Capella and Procyon lines of sight.

The $\mathrm{H}$ i column density listed in Table 4 for the $\lambda$. And line of sight, $\log \mathrm{N}_{\mathrm{HI}}=18.45 \pm 0.15$, indicates an average density of $n_{\mathrm{HI}}=0.040 \pm 0.014 \mathrm{~cm}^{-3}$. This density is much lower than that found for the shorter $(d<4 \mathrm{pc})$ lines of sight toward $\alpha$ Cen, Procyon, and $\epsilon$ Ind, but it is very similar to the density measured toward Capella $\left(d=12.5 \mathrm{pc} ; n_{\mathrm{H} I}=\right.$ $0.0450 \pm 0.0006 \mathrm{~cm}^{-3}$ ). In Paper II, this difference was interpreted to mean that most of the Capella line of sight is not inside the local cloud but is instead within a very hot $\left(T \approx 10^{6} \mathrm{~K}\right)$ and tenuous phase of the ISM containing essentially no neutral hydrogen. This is a reasonable hypothesis, since the Sun is believed to be located within a region called the Local Bubble, in which most of the volume of the bubble consists of this very hot interstellar matter. It is this hot interstellar material that is thought to be responsible for the soft X-ray background (see, e.g., Cox \& Reynolds 1987). This interpretation of the Capella data is supported by Lemoine et al. (1995), who measured an identical H I column density for this absorption component along a much longer line of sight only 7 from Capella (see $\$ 1$ ). If the local cloud extended to and beyond Capella, Lemoine et al. (1995) should have measured a larger column density than that measured toward Capella.

Does the low average $\mathrm{H}$ I density toward $\lambda$ And mean that $\lambda$ And lies inside the very hot, $10^{6} \mathrm{~K}$ phase of the ISM? We believe that this is unlikely, because hydrogen walls such as the one we believe we have detected around $\lambda$ And cannot exist unless the surrounding interstellar material contains a substantial amount of neutral hydrogen. Thus, although it is likely that a portion of the $\lambda$ And line of sight lies within the hot phase of the ISM, $\lambda$ And itself must lie within a warm, neutral cloud like that which surrounds the Sun. It is also possible that the low ISM density we have measured toward $\lambda$ And is simply indicative of density gradients in the local cloud (and the cloud surrounding $\lambda$ And, if it is a different cloud) rather than the presence of very hot and completely ionized ISM material along this line of sight.

We find that $\mathrm{D} / \mathrm{H}=(1.7 \pm 0.5) \times 10^{-5}$ for the $\lambda$ And data. Both the $\epsilon$ Ind and $\lambda$ And data appear to support the value of $\mathrm{D} / \mathrm{H}=1.6 \times 10^{-5}$ established in Papers I-III as the best estimate for the LISM. However, we consider the $\epsilon$ Ind result to represent a more significant confirmation of this valuc, because the bisector method used to constrain possible values of $N_{\mathrm{HI}}$ for $\epsilon$ Ind is a quantitative method that produces a clear result. In contrast, the method used to constrain $N_{\mathrm{H}}$ for $\lambda$ And relies on qualitative assumptions, namely the requirement that the second absorption component be interpretable as a hydrogen-wall component. The analyses in Papers I-III clearly show that the abundance ratio $\mathrm{D}_{\mathrm{I}} / \mathrm{Mg}$ il does vary significantly in the LISM, but difficulties in accurately measuring $\mathrm{H}_{1}$ column densities have prevented us from determining whether it is D I or $\mathrm{Mg}$ II or both that are varying. Therefore, there is as yet no conclusive evidence from the GHRS studies of many lines of sight that $\mathrm{D} / \mathrm{H}$ varies in the LISM, as many analyses of $I U E$ and Copernicus data have suggested (see, e.g., Duprce, Baliunas, \& Shipman 1977).

\section{THE HYDROGEN WALLS SURROUNDING $\epsilon$ INDI AND $\lambda$ ANDROMEDAE}

To understand the properties of the hydrogen walls that we believe we have detected around $\epsilon$ Ind and $\lambda$ And, it is necessary to evaluate the velocity and direction of the interstellar flow in the rest frames of these stars. We assume that $\epsilon$ Ind is in the G cloud and that $\lambda$ And is in the AG cloud. As discussed in $\S 4.1$, it is possible that $\epsilon$ Ind is actually in the AG cloud, but since the $V_{\mathrm{G}}$ and $V_{\mathrm{AG}}$ vectors are very similar (see $\$ 1$ ), it does not matter too much which vector we use. The use of the $V_{A G}$ vector to represent the ISM flow around $\lambda$ And is more questionable. At a distance of $23 \mathrm{pc}, \lambda$ And likely lies well outside the AG cloud. However, since the velocity of the interstellar absorption agrees very well with the velocity expected from the $V_{A G}$ vector, the flow vector for the cloud in which $\lambda$ And resides is probably similar to the $V_{\mathrm{AG}}$ vector

The distances, radial velocities $\left(v_{r}\right)$, and proper motions ( $\mu_{x}$ and $\mu_{\delta} ;$ Hirshfeld, Sinnott, \& Ochsenbein 1991) of $\epsilon$ Ind and $\lambda$ And are listed in Table 5 . When combined with the flow vectors assumed above and the known positions of the stars, this information allows us to compute the interstellar flow vectors in the rest frames of the stars. The velocities $\left(l_{0}\right)$ and directions $(l$ and $b)$ of these flow vectors are also listed in Table 5. These flow vectors may be compared with the $V_{\mathrm{AG}}$ vector $\left(v_{0}=25.7 \mathrm{~km} \mathrm{~s}^{-1}, l=186.1, b=-16.4\right)$ that represents the interstellar flow in the solar rest frame. The interstellar wind velocity in the stellar rest frame $v_{0}$, is much higher for $\epsilon$ Ind and $\lambda$ And than it is for the Sun. We believe that this is the reason that the hydrogen walls of $\epsilon$ Ind and $\lambda$ And have higher temperatures than the solar hydrogen 
TABLE 5

Interstellar Wind Properties in the Rest Frames of $\epsilon$ INd and $\lambda$ And

\begin{tabular}{|c|c|c|c|c|c|c|c|c|c|}
\hline Star & Cloud & $\begin{array}{l}\text { Distance } \\
\text { (pc) }\end{array}$ & $\left(\mathrm{km} \mathrm{s}^{\mathrm{v}_{r}}\right)$ & $\frac{\mu_{x}}{\left(\operatorname{arcsec} y r^{-1}\right)}$ & $\begin{array}{c}\mu_{\delta} \\
\left(\operatorname{arcsec} \mathrm{yr}^{-1}\right)\end{array}$ & $\left(\mathrm{km} \mathrm{s}^{-1}\right)$ & $\begin{array}{c}l \\
(\mathrm{deg})\end{array}$ & $\begin{array}{c}b \\
\text { (deg) }\end{array}$ & $\begin{array}{c}\theta \\
\text { (deg) }\end{array}$ \\
\hline$\epsilon$ Ind $\ldots \ldots$ & $\mathrm{G}$ & 3.54 & -38.9 & +3.96 & -2.54 & 64.0 & 36.2 & -13.4 & 60.3 \\
\hline$\lambda$ And $\ldots .$. & $\mathrm{AG}$ & 23 & +6.8 & +0.17 & -0.42 & 47.7 & 169.4 & +61.6 & 88.9 \\
\hline
\end{tabular}

wall. Figure 12 shows the hydrogen-wall temperatures as a function of interstellar wind velocity. Baranov \& Malama (1995) and Pauls et al. (1995) predict a temperature of about $20,000 \mathrm{~K}$ for the solar hydrogen wall. V. B. Baranov (private communication) informs us that if the Sun were traveling through the ISM at a velocity of $64.0 \mathrm{~km} \mathrm{~s}^{-1}$ like $\epsilon$ Ind, his models would predict a hydrogen-wall temperature of about $80,000 \mathrm{~K}$ rather than $20,000 \mathrm{~K}$. Thus, the dependence of temperature on wind velocity seen in Figure 12 is qualitatively consistent with these models.

One might think that hydrogen should be almost completely ionized at these high temperatures. However, it is not collisions that heat neutral hydrogen but charge exchange processes. Interstellar protons are compressed and heated by their collisions with solar wind protons. Through charge exchange with these protons, the neutral hydrogen is also compressed and heated, creating the hot hydrogen wall. Since the collisional ionization mean free path for hydrogen atoms at very low ISM densities is extremely long $(\approx 0.1 \mathrm{pc})$. hydrogen atoms heated in the hydrogen wall are not collisionally ionized there despite the high temperatures.

On the basis of the models of the heliosphere, we expect the solar hydrogen wall to have different properties for different lines of sight. In Paper III, the solar hydrogen wall was observed toward $\alpha \mathrm{Cen}$ at an angle of $\theta=52$ from the upwind direction. The last column of Table 5 lists the angles (relative to the upwind direction as seen from the star) observed through the hydrogen walls of $\epsilon$ Ind and $\lambda$ And. Fortunately, these angles $\left(60^{\circ}\right.$ and $\left.89^{\circ}\right)$ are not drastically different from the solar angle observed in Paper III, allowing us to compare results as we do in Figure 12.

The column density of the $\lambda$ And hydrogen wall, $\log \mathrm{N}_{\mathrm{HI}}=$ $14.8 \pm 0.2$, is very similar to the column density measured for the solar hydrogen wall in Paper III $\left(\log \mathrm{N}_{\mathrm{HI}}=14.74\right.$

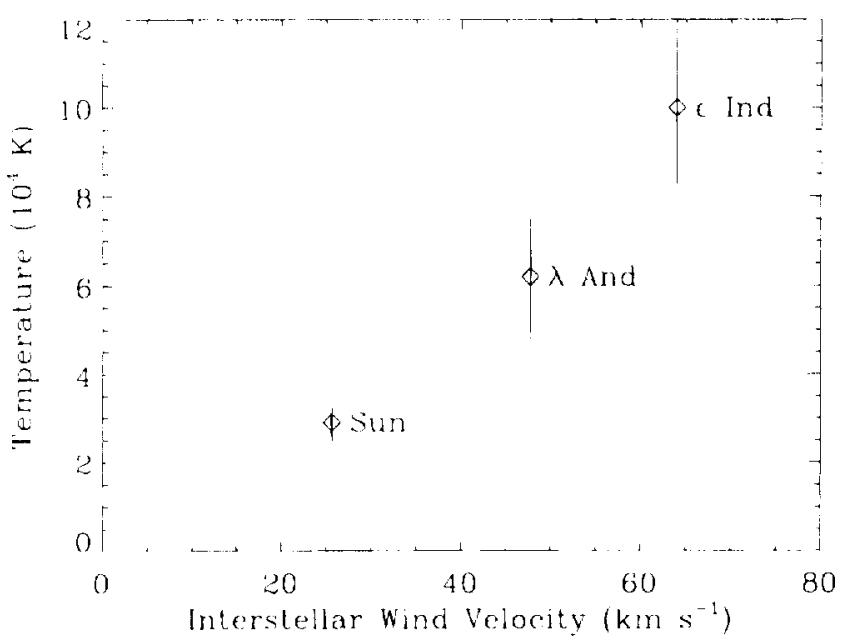

FIG. 12.-Hydrogen-wall temperature as a function of the interstellar wind velocity in the stellar rest frame. $\pm 0.24)$. The hydrogen wall column density for $\epsilon$ Ind, however, is much lower- $\log \mathrm{N}_{\mathrm{HI}}=14.2 \pm 0.2$. Perhaps the high interstellar wind velocity for $\epsilon$ Ind has resulted in a thinner hydrogen wall.

We expect the hydrogen walls to generally have velocities between the stellar radial velocity and the velocity of the ISM absorption, because the hydrogen-wall material is expected to be decelerated relative to the unperturbed interstellar wind. Baranov \& Malama (1993) predict that $v_{z} \approx$ $0.5 v_{0}$ in the upwind (i.e., $\theta=0$ ) direction and $v_{z} \approx 0.75 v_{0}$ at $\theta=90^{\circ}$, where $v_{z}$ is the velocity component in the direction of the unperturbed flow. Away from the upwind direction, the hydrogen-wall material can also have a velocity component, $v_{x}$, perpendicular to the original flow direction as the interstellar flow is deflected around the heliopause (see Fig. 1). For $\theta=90$, Baranov \& Malama (1993) predict that $v_{x} \approx 0.2 v_{0}$.

To estimate the expected hydrogen wall velocity for $\epsilon$ Ind, we crudely interpolate the results of Baranov \& Malama quoted above to $\theta=60$ and assume $v_{z}=0.65 v_{0}$ and $v_{x}=$ $0.15 v_{0}$. The expected velocity is then simply $v=v_{r}+v_{z}$ $\cos \theta-v_{x} \sin \theta=-27 \mathrm{~km} \mathrm{~s}^{-1}$. This velocity agrees perfectly with the measured velocity in Table 4 of $-27 \pm 6 \mathrm{~km}$ $\mathrm{s}^{-1}$. Since $\theta$ is essentially 90 for $\lambda$. And, the $v_{z}$ velocity component has no projection along the line of sight, and therefore it is the $v_{x}$ component that is responsible for the observed blueshift of the hydrogen wall with respect to the interstellar absorption and the stellar radial velocity noted in $\S 4.2$. For $\lambda$ And, the predicted velocity is $-3 \mathrm{~km} \mathrm{~s}^{-1}$, which agrees well with the measured velocity listed in Table $4\left(-2 \pm 3 \mathrm{~km} \mathrm{~s}^{-1}\right)$.

Because the parameters of the hot components seen toward $\epsilon$ Ind and $\lambda$ And agree very well with the expected properties of hydrogen walls around these stars, we believe the hydrogen wall interpretation is the best interpretation for the hot components. Nevertheless, there is no way we can prove that the material responsible for the absorption in these components is in fact surrounding the stars. Other interpretations of the hot components cannot be completely ruled out. For example, the local warm neutral cloud is embedded within a very hot $\left(\approx 10^{6} \mathrm{~K}\right)$, tenuous ISM. If there is an evaporative interface between the local cloud and this hot ISM (see, e.g., Slavin 1989) along the lines of sight to $\epsilon$ Ind and $\lambda$ And, the hot components might possibly be associated with this interface. For the line of sight toward Sirius, Bertin et al. (1995) claim to have detected a hot $\mathrm{H} \mathrm{I}$ absorption component that is redshifted relative to the interstellar absorption. They propose that this absorption component is due to an evaporation flow from the local cloud into the hot ISM at an evaporative interface along this line of sight. We point out, however, that the hot components seen toward $\epsilon$ Ind and $\lambda$ And are definitely blueshifted relative to the interstellar absorption, making it much harder to associate these components with an evaporative interface. 


\section{THE STELLAR WINDS OF $\epsilon$ INDI AND $\lambda$ ANDROMEDAE}

In $\S 4.2$, we noted that the presence of neutral hydrogen in the ISM is necessary for the formation of a hydrogen wall, meaning $\epsilon$ Ind and $\lambda$ And must be inside warm, neutral clouds. Another necessary ingredient for the formation of hydrogen walls is a stellar wind, the implication being that $\epsilon$ Ind and $\lambda$ And must have previously undetected winds. To our knowledge, no one has ever detected a solar-like wind around a star before. Other types of stellar winds have been detected and studied extensively: for example, the hot winds of OB stars that are driven by radiation pressure, the cool winds of red giant and supergiant stars, and the winds of pre-main-sequence stars such as $\mathrm{T}$ Tauri and Herbig Ae stars. However, all these winds are fundamentally different from the solar wind in many ways. The wind acceleration mechanisms are almost certainly different, and the massloss rates of these winds are orders of magnitude higher than for the solar wind. Also, the stars generating these winds are very different from the Sun.

On the basis of what is known about the origins of the solar wind, there is no reason to believe that stars like $\epsilon$ Ind and $\lambda$ And do not have similar winds. In the original solar wind model of Parker (1958), the solar wind is driven by the thermal expansion of material heated to coronal temperatures $\left(T \approx 2 \times 10^{6} \mathrm{~K}\right)$. Therefore, all stars that have hot coronae are expected to have solar-like winds. Coronal $\mathrm{X}$-ray emission has been detected from both $\epsilon$ Ind and $\lambda$ And. The X-ray luminosity of $\epsilon$ Ind $\left(L_{X}=1.6 \times 10^{27}\right.$ ergs $\mathrm{s}^{-1}$; Wood et al. 1994) is very similar to that of the Sun, implying that $\epsilon$ Ind and the Sun have similar coronal properties. The X-ray luminosity of $\lambda$ And, on the other hand $\left(L_{\mathrm{X}}=3.7 \times 10^{30} \mathrm{ergs} \mathrm{s}^{-1}\right.$; Dempsey et al. 1993a), is over 1000 times larger than that of the Sun. The temperature of $\lambda$ And's corona is also significantly higher (Dempsey et al. 1993b), implying a much more active corona than the Sun, and perhaps different wind properties.

Detecting a solar-like wind emanating from a star is much more difficult than detecting the corona that presumably provides the acceleration mechanism. Hot, ionized winds should be sources of free-free emission, so nondetections of radio emission from stars have allowed upper limits to be placed on the mass-loss rates of these stars. For example, Brown et al. (1990) list upper limits for mass-loss rates of many A- and F-type stars, the lowest upper limit being $\dot{M}<7 \times 10^{-11} M_{\odot} \mathrm{yr}^{-1}$ for Procyon (F5 IV-V). Lim, White, \& Slee (1996) place an upper limit of $\dot{M}<7$ $\times 10^{-12} M_{\odot} \mathrm{yr}^{-1}$ on the mass-loss rate of Proxima Cen (M5.5 Ve). However, these upper limits are more than 2 orders of magnitude higher than the solar mass-loss rate of $\dot{M}=2 \times 10^{-14} M_{\odot} \mathrm{yr}^{-1}$ (Feldman et al. 1977), illustrating just how difficult it is to detect such winds. The creation of "hydrogen walls" when these winds collide with the ISM provides indirect evidence for such winds. With the detection of hydrogen walls around $\epsilon$ Ind and $\lambda$ And, perhaps we can now add the solar wind to the long list of solar phenomena that have been detected on other stars.

\section{SUMMARY}

We have observed the Ly $\alpha$ lines of $\epsilon$ Ind and $\lambda$ And using the GHRS instrument aboard the HST. Our analysis of the interstellar absorption features present in these lines reveals that the velocities and temperatures of the $D_{I}$ lines appear to be different from those of the $\mathrm{H}$ I lines. A similar discrep- ancy was seen in Paper III for the Ly $\alpha$ lines of $\alpha$ Cen A and B. In Paper III, the discrepancy was resolved by fitting the H I line with two components, one representing absorption from the ISM and the other representing absorption from the hydrogen wall surrounding the Sun. This hydrogen wall has been predicted by models of the heliospheric interface region where the solar wind interacts with the ISM.

We have also fitted the $H \mathrm{I}$ lines of $\epsilon$ Ind and $\lambda$ And with two components in order to resolve the discrepancy between $\mathrm{D}_{\mathrm{I}}$ and $\mathrm{H}$ I. The results of these two-component fits are summarized in Table 4. We found, however, that the parameters of the second components for $\epsilon$ Ind and $\lambda$ And were inconsistent with the solar hydrogen wall. The temperatures are too high, and for $\epsilon$ Ind the velocity is clearly wrong. However, the parameters of these second components are consistent with those expected for hydrogen walls surrounding the stars. The hydrogen walls of $\epsilon$ Ind and $\lambda$ And are expected to be hotter than the solar hydrogen wall because of the higher interstellar wind velocities in the stellar rest frames. In recent models of the heliospheric interface region, hydrogen walls are a natural consequence of the interaction between an ionized wind and a partially ionized interstellar medium. Thus, the detection of hydrogen walls around $\epsilon$ Ind and $\lambda$ And represents a detection of winds emanating from these stars. We believe this to be the first detection, albeit indirect, of solar-like winds around stars other than the Sun.

As for the measured parameters of the LISM listed in Table 4, the $\mathrm{H}$ I column density measured for the $\epsilon$ Ind line of sight implies an average density, $n_{\mathrm{HI}}=0.094 \pm 0.022$ $\mathrm{cm}^{-3}$, that is consistent with previous measurements made for other short $(d<4 \mathrm{pc})$ lines of sight. The H I column density was not measured by the two-component fit alone. Instead, we used a promising new technique for estimating $N_{\mathrm{H} \text { I }}$ that is feasible when there is a large velocity separation between the stellar Ly $\alpha$ emission line and the interstellar absorption. The advantage of this technique is that it does not require one to estimate the shape of the intrinsic stellar Ly $\alpha$ profile. This technique could not be used for $\lambda$ And, but our requirement that the second component of the twocomponent fits be interpretable as either a solar or stellar hydrogen wall component resulted in substantial constraints on the shape of the assumed Ly $\alpha$ profile and on $N_{\mathrm{H}}$ I (see Table 4).

The column density we have measured toward $\lambda$ And implies a LISM density, $n_{\mathrm{H} \text { I }}=0.040 \pm 0.014 \mathrm{~cm}^{-3}$, that is lower than densities measured along much shorter lines of sight, implying either a density gradient in the LISM or very hot, ionized ISM material along much of this line of sight. The LISM temperature listed for the $\lambda$ And line of sight in Table $4, T=11,500 \pm 500 \mathrm{~K}$, is significantly higher than previous measurements for the LISM. Considering the length of this line of sight, this measurement could be the result of multiple ISM components rather than high temperatures. Observations of narrower interstellar absorption lines, like those of $\mathrm{Mg}$ II and Fe II used in Papers I-II, are needed to detect multiple components. If future observations prove that multiple ISM cmponents exist for the $\lambda$ And line of sight, the $\mathrm{Ly} \alpha$ line of $\lambda$ And should be reanalyzed with this in mind, and the existence of the hydrogen wall absorption component for this line of sight should be reassessed. The $\mathrm{D} / \mathrm{H}$ measurements listed for $\epsilon$ Ind and $\lambda$ And in Table 4 agree very well with the value of $\mathrm{D} / \mathrm{H}=$ $1.6 \times 10^{-5}$ established in Papers I-III as the best esti- 
mate for $\mathrm{D} / \mathrm{H}$ in the LISM. Thus, the conclusions in Papers I-II regarding this value and its implications for cosmology remain valid.

We would like to thank V. B. Baranov for providing us with useful information about the models of the heliospheric interface region, and F. Bruhweiler for helpful sug- gestions. This work is supported by NASA Interagency Transfer S-56460-D to the National Institute of Standards and Technology. W. R. A. also acknowledges the support of NASA grant GO-0100.01-92A from the Space Telescope Science Institute, which is operated by the Association of Universities for Research in Astronomy Inc., under NASA contract NAS 5-26555.
Baliunas, S. L., \& Dupree, A. K. 1979, ApJ, 227,870

Baranov, V. B., \& Malama, Y. G. 1993, J. Geophys. Res., 98, 15,157 1995, J, Geophys. Res., 100, 14,755

Batten, A. H., Fletcher, J. M., \& Mann, P. J. 1978, Publ. Dom. Astrophys. Obs. Victoria, 15, No. 5

Bertin, P., Vidal-Madjar, A., Lallement, R., Ferlet, R., \& Lemoine, M. 1995, A\&A, 302, 889

Bevington, P. R., \& Robinson, D. K. 1992, Data Reduction and Error Analysis for the Physical Sciences (New York: McGraw-Hill)

Brown, A., Vealé, A., Judge, P., Bookbinder, J. A., \& Hubeny, I. 1990, ApJ, 361,220

Buscombe, W., \& Kennedy, P. M. 1968, MNRAS, 139, 341

Cox. D. P., \& Reynolds, R. J. 1987, ARA\&\&A, 25, 203

Dempsey, R. C., Linsky, J. L., Fleming, T. A., \& Schmitt, J. H. M. M. 1993a, ApJS, 86, 599

Dempsey, R. C., Linsky, J. L., Schmitt, J. H. M. M., \& Fleming T. A. 1993b, ApJ, 413,333

Dupree, A. K., Baliunas, S. L., \& Shipman, H. L. 1977, ApJ, 218, 36

Feldman, W. C., Asbridge, J. R., Bame, S. J., \& Gosling, J. T. 1977, in The Solar Output and Its Variations, ed. O. R. White (Boulder: Colorado Assoc. Univ. Press), 351

Ferlet, R., Lecavelier, A., Vidal-Madjar, A., Bertin, P., Deleuil, M., Gry, C., Langrange-Henry, A.-M., \& Lallement, R. 1995, A\&A, 297, L5

Gilliland, R. L. 1994, GHRS Instrument Science Report 063 (Baltimore Space Telecope Science Instutute)

Gliese, W., \& Jahreiss. H. 1991, Third Catalogue of Nearby Stars (Veröfentlichungen des Astronomischen Rechen-Instituts, University of Heidelberg)

Gry, C., Lemonon, L., Vidal-Madjar, A., Lemoine, M., \& Ferlet, R. 1995, A\&A, 302,497

Herbst, W. 1973, A\&A, 26, 137

Hirshfeld, A., Sinnott, R. W., \& Ochsenbein, F. 1991, Sky Catalogue 2000.0, Vol. 1 (Cambridge: Cambridge Univ. Press)

Jenkins, L. F. 1952, General Catalog of Trigonometric Parallaxes (New Haven: Yale Obs.)

\section{EFERENCES}

Lallement, R., \& Bertin, P. 1992, A\&A, 266, 479

Lallement, R., Bertin, P., Ferlet, R., Vidal-Madjar, A., \& Bertaux, J. L. 1994, A\&A, 286, 898

Lallement, R., Ferlet, R., Lagrange, A. M., Lemoine, M., \& Vidal-Madjar, A. 1995, A\&A, 304, 461

Lemoine, M., Vidal-Madjar, A., Ferlet, R., Bertin, P., Gry, C., \& Lallement, R. 1995, in The Light Element Abundances, ed. P. Crane (Berlin: Springer), 233

Lim, J. White, S. M., \& Slee, O. B. 1996, ApJ, 460, 976

Linsky, J. L., et al. 1993, ApJ, 402, 694 (Paper I)

Linsky, J. L., Diplas, A. Wood, B. E., Brown, A., Ayres, T. R., \& Savage, B. D. 1995, ApJ, 451, 335 (Paper II)

Linsky, J. L., \& Wood, B. E. 1996, ApJ, 463, 254 (Paper III)

McClintock, W., Henry, R. C., Linsky, J. L., \& Moos, H. W. 1978, ApJ, 225, 465

Morton, D. C. 1991, ApJS, 77, 119

Murthy, J., Henry, R. C., Moos, H. W., Vidal-Madjar, A., Linsky, J. L., \& Gry, C. 1990 , ApJ, 356, 223

Parker, E. N. 1958, ApJ, 128, 664

Pauls, H. L., Zank, G. P., \& Williams, L. L. 1995, J. Geophys. Res., 100, 21,595

Slavin, J. 1989, ApJ, 346, 718

Strassmeier, K. G., Hall, D. S., Zeilik, M., Nelson, E., Eker, Z., \& Fekel, F. C. 1988, A\&AS, 72, 291

Walker, T. P., Steigman, G., Schramm, D. N., Olive, K. A., \& Kang. H.-S 1991, ApJ, 376, 51

Witte, M., Rosenbeuer, H., Banaszkewicz, M., \& Fahr, H. 1993, Adv. Space Res., 13(6), 121

Wood, B. E., Brown, A., Linsky, J. L., Kellett, B. J., Bromage, G. E., Hodgkin, S. T., \& Pye, J. P. 1994, ApJS, 93, 287

Wood, B.E., Harper, G. M., Linsky, J. L., \& Dempsey, R. C. 1996, ApJ, 458, 761 
